\title{
Adaptive Exponential Synchronization for Stochastic Competitive Neural Networks with Time-Varying Leakage Delays and Reaction-Diffusion Terms
}

\author{
Zhiqiang Wang, ${ }^{1,2}$ Lili Wang, ${ }^{3}$ and Rui $\mathrm{Xu}^{3}$ \\ ${ }^{1}$ Institute of Applied Mathematics, Hebei Academy of Sciences, No. 46 South Youyi Street, Shijiazhuang 050081, China \\ ${ }^{2}$ Hebei Authentication Technology Engineering Research Center, No. 46 South Youyi Street, Shijiazhuang 050081, China \\ ${ }^{3}$ Institute of Applied Mathematics, Shijiazhuang Mechanical Engineering College, No. 97 West Heping Road, \\ Shijiazhuang 050003, China
}

Correspondence should be addressed to Zhiqiang Wang; wangzhiqiang@heb-math.org

Received 20 February 2017; Accepted 2 May 2017; Published 20 August 2017

Academic Editor: Marco Mussetta

Copyright (C) 2017 Zhiqiang Wang et al. This is an open access article distributed under the Creative Commons Attribution License, which permits unrestricted use, distribution, and reproduction in any medium, provided the original work is properly cited.

We study the exponential synchronization problem for a class of stochastic competitive neural networks with different timescales, as well as spatial diffusion, time-varying leakage delays, and discrete and distributed time-varying delays. By introducing several important inequalities and using Lyapunov functional technique, an adaptive feedback controller is designed to realize the exponential synchronization for the proposed competitive neural networks in terms of $p$-norm. According to the theoretical results obtained in this paper, the influences of the timescale, external stimulus constants, disposable scaling constants, and controller parameters on synchronization are analyzed. Numerical simulations are presented to show the feasibility of the theoretical results.

\section{Introduction}

Neural networks are mathematical models that are inspired by the structure and functional aspects of biological neural networks. Meyer-Baese et al. [1] proposed competitive neural networks with different timescales, which describe the dynamics of cortical cognitive maps with unsupervised synaptic modifications. In the competitive neural networks model, there are two types of state variables: the short-termmemory (STM) variables describing the fast neural activity and the long-term-memory (LTM) variables describing the slow unsupervised synaptic modifications. Hence, there are two timescales in the competitive neural networks, one of which corresponds to the fast change of the state and the other to the slow change of the synapse by external stimuli. The above competitive neural networks are described by the following differential equations:

$$
\begin{aligned}
\text { STM: } \varepsilon \frac{\mathrm{d} y_{i}(t)}{\mathrm{d} t}= & -c_{i} y_{i}(t)+\sum_{j=1}^{n} a_{i j} f\left(y_{j}(t)\right) \\
& +H_{i} \sum_{l=1}^{r} m_{i l}(t) w_{l}, \\
\text { LTM: } \frac{\mathrm{d} m_{i l}(t)}{\mathrm{d} t}= & -m_{i l}(t)+w_{l} f\left(y_{i}(t)\right),
\end{aligned}
$$

where $i=1,2, \ldots, n, y_{i}(t)$ is the neuron current activity level, $m_{i l}(t)$ is the synaptic efficiency, $f\left(y_{j}(t)\right)$ is the output of neurons, $c_{i}>0$ is the time constant of the neuron, $a_{i j}$ denotes the connection strength of the $j$ th neuron on the $i$ th neuron, $H_{i}$ is the strength of the external stimulus, $w_{l}$ is the constant external stimulus, $r$ is the number of the constant external stimuli, and $\varepsilon>0$ is the timescale of the STM state. 
Synchronization problems of neural networks have been widely researched because of their extensive applications in secure communication, information processing, and chaos generators design. Synchronization of competitive neural networks with different timescales has attracted a great interest [2-7]. In [7], Gan et al. studied the adaptive synchronization for a class of competitive neural networks with different timescales and stochastic perturbation by constructing a Lyapunov-Krasovskii functional:

$$
\begin{aligned}
\text { STM: } \varepsilon \frac{\mathrm{d} y_{i}(t)}{\mathrm{d} t}= & -c_{i} y_{i}(t)+\sum_{j=1}^{n} a_{i j} f_{j}\left(y_{j}(t)\right) \\
& +\sum_{j=1}^{n} b_{i j} f_{j}\left(y_{j}(t-\tau(t), x)\right) \\
& +\sum_{j=1}^{n} d_{i j} \int_{t-\tau^{*}(t)}^{t} f_{j}\left(y_{j}(s)\right) \mathrm{d} s \\
& +H_{i} \sum_{l=1}^{r} m_{i l}(t) w_{l}, \\
\text { LTM: } \frac{\mathrm{d} m_{i l}(t)}{\mathrm{d} t}= & -K_{i} m_{i l}(t)+w_{l} f_{i}\left(y_{i}(t)\right),
\end{aligned}
$$

where $\tau(t)$ and $\tau^{*}(t)$ are the discrete time-varying delay and the distributed time-varying delay, respectively; $b_{i j}$ and $d_{i j}$ are, respectively, the discrete time-varying delay connection strength and the distributed time-varying delay connection strength of the $j$ th neuron on the $i$ th neuron; $K_{i}$ is the disposable scaling constant.

The first term $y_{i}(t)$ in each of the right sides of (2) is called leakage term corresponding to a stabilizing negative feedback of the system $[8,9]$. In real world, the transmission delays often appear in leakage terms, which are called leakage delays [10]. It is well known that leakage delays have been incorporated into neural networks by many researchers [1114]. However, leakage delays of neural networks in most bibliographies listed above are constants. As pointed out in [15-18], the delays in neural networks are usually timevarying. Hence, the results about the neural networks with constant delays in the leakage term are imperfect.

In addition, dynamic behaviors of neural networks derive from the interactions of neurons, which is dependent on not only the time of each neuron but also its space position $[19,20]$. From this point, diffusion phenomena should not be ignored in neural networks. Many good results about reaction-diffusion neural networks have been obtained [2125]. The boundary conditions in most literatures listed are assumed to be Dirichlet boundary conditions. In engineering applications, such as thermodynamics, Neumann boundary conditions need to be considered. As far as we know, there are few results concerning the synchronization of competitive neural networks with reaction-diffusion term under Neumann boundary conditions.

Based on the above discussion, we are concerned with the combined effects of time-varying leakage delays, stochastic perturbation, and spatial diffusion on the synchronization of competitive neural networks with Neumann boundary conditions in terms of $p$-norm via an adaptive feedback controller to improve the previous results. To this end, we discuss the following neural networks:

$$
\begin{aligned}
\text { STM: } \varepsilon \frac{\partial y_{i}(t, x)}{\partial t}= & D_{i} \Delta y_{i}(t, x)-c_{i} y_{i}\left(t-\delta_{i}(t), x\right) \\
& +\sum_{j=1}^{n} a_{i j} f_{j}\left(y_{j}(t, x)\right) \\
& +\sum_{j=1}^{n} b_{i j} f_{j}\left(y_{j}\left(t-\tau_{i j}(t), x\right)\right) \\
& +\sum_{j=1}^{n} d_{i j} \int_{t-\tau_{i j}^{*}(t)}^{t} f_{j}\left(y_{j}(s, x)\right) \mathrm{d} s \\
& +H_{i} \sum_{l=1}^{r} m_{i l}(t, x) w_{l}, \\
\operatorname{LTM}: \frac{\partial m_{i l}(t, x)}{\partial t}= & -K_{i} m_{i l}(t, x)+w_{l} f_{i}\left(y_{i}(t, x)\right),
\end{aligned}
$$

where $x=\left(x_{1}, x_{2}, \ldots, x_{m}\right)^{T} \in \Omega \subset \mathbb{R}^{m}$ and $\Omega=\left\{x=\left(x_{1}, x_{2}\right.\right.$, $\left.\left.\ldots, x_{m}\right)^{T}|| x_{k} \mid<m_{k}\right\}$ is a bound compact set with smooth boundary $\partial \Omega$ and mes $\Omega>0$ in space $\mathbb{R}^{m} ; y(t, x)=\left(y_{1}(t, x)\right.$, $\left.y_{2}(t, x), \ldots, y_{n}(t, x)\right)$ with $y_{i}(t, x)$ denotes the state of the $i$ th neuron at time $t$ and in space $x ; \Delta=\sum_{k=1}^{m}\left(\partial^{2} / \partial x_{k}^{2}\right)$ is the Laplace operator; $0<\tau_{i j}(t) \leq \tau$ and $0<\tau_{i j}^{*}(t) \leq \tau^{*}$ are the discrete time-varying delay and the distributed time-varying delay, respectively; $0<\delta_{i}(t) \leq \delta$ is the time-varying leakage delay; $D_{i}>0$ corresponds to the transmission diffusion coefficient along the $i$ th neuron.

Let $s_{i}(t, x)=\sum_{l=1}^{r} m_{i l}(t, x) w_{l}=m_{i}^{T}(t, x) w$, where $m_{i}(t, x)=\left(m_{i 1}(t, x), m_{i 2}(t, x), \ldots, m_{i r}(t, x)\right)^{T}$ and $w=\left(w_{1}\right.$, $\left.w_{2}, \ldots, w_{r}\right)^{T}$, and then then system (3) can be rewritten as

$$
\begin{aligned}
\text { STM: } \varepsilon \frac{\partial y_{i}(t, x)}{\partial t}= & D_{i} \Delta y_{i}(t, x)-c_{i} y_{i}\left(t-\delta_{i}(t), x\right) \\
& +\sum_{j=1}^{n} a_{i j} f_{j}\left(y_{j}(t, x)\right) \\
& +\sum_{j=1}^{n} b_{i j} f_{j}\left(y_{j}\left(t-\tau_{i j}(t), x\right)\right) \\
& +\sum_{j=1}^{n} d_{i j} \int_{t-\tau_{i j}^{*}(t)}^{t} f_{j}\left(y_{j}(s, x)\right) \mathrm{d} s \\
& +H_{i} s_{i}(t, x), \\
\text { LTM: } \frac{\partial s_{i}(t, x)}{\partial t}= & -K_{i} s_{i}(t, x)+|w|^{2} f_{i}\left(y_{i}(t, x)\right),
\end{aligned}
$$

where $|w|^{2}=w_{1}^{2}+w_{2}^{2}+\cdots+w_{r}^{2}$. Without loss of generality, the input stimulus vector $w$ is assumed to be normalized with 
magnitude $|w|^{2}=1$. System (4) is simplified to

STM: $\varepsilon \frac{\partial y_{i}(t, x)}{\partial t}=D_{i} \Delta y_{i}(t, x)-c_{i} y_{i}\left(t-\delta_{i}(t), x\right)$

$$
\begin{aligned}
& +\sum_{j=1}^{n} a_{i j} f_{j}\left(y_{j}(t, x)\right) \\
& +\sum_{j=1}^{n} b_{i j} f_{j}\left(y_{j}\left(t-\tau_{i j}(t), x\right)\right) \\
& +\sum_{j=1}^{n} d_{i j} \int_{t-\tau_{i j}^{*}(t)}^{t} f_{j}\left(y_{j}(s, x)\right) \mathrm{d} s \\
& +H_{i} s_{i}(t, x),
\end{aligned}
$$

LTM: $\frac{\partial s_{i}(t, x)}{\partial t}=-K_{i} s_{i}(t, x)+f_{i}\left(y_{i}(t, x)\right)$.

The boundary condition of system (5) takes the form

$$
\begin{aligned}
& \frac{\partial y_{i}(t, x)}{\partial \mathbf{n}}:=\left(\frac{\partial y_{i}(t, x)}{\partial x_{1}}, \frac{\partial y_{i}(t, x)}{\partial x_{2}}, \ldots, \frac{\partial y_{i}(t, x)}{\partial x_{m}}\right)^{T} \\
&=\mathbf{0}, \quad(t, x) \in[-\bar{\tau},+\infty) \times \partial \Omega, \\
& \frac{\partial s_{i}(t, x)}{\partial \mathbf{n}}::\left(\frac{\partial s_{i}(t, x)}{\partial x_{1}}, \frac{\partial s_{i}(t, x)}{\partial x_{2}}, \ldots, \frac{\partial s_{i}(t, x)}{\partial x_{m}}\right)^{T}=\mathbf{0}, \\
&(t, x) \in[-\bar{\tau},+\infty) \times \partial \Omega .
\end{aligned}
$$

The initial value of system (5) takes the form

$$
\begin{aligned}
& y(\theta, x)=\phi^{y}(\theta, x), \\
& s(\theta, x)=\phi^{s}(\theta, x),
\end{aligned}
$$

$$
(\theta, x) \in[-\bar{\tau}, 0] \times \Omega,
$$

where $\bar{\tau}=\max \left\{\delta, \tau, \tau^{*}\right\}, s(\theta, x)=\left(s_{1}(\theta, x), s_{2}(\theta, x)\right.$, $\left.\ldots, s_{n}(\theta, x)\right)^{T}, \phi^{y}(\theta, x)=\left(\phi_{1}^{y}(\theta, x), \phi_{2}^{y}(\theta, x), \ldots, \phi_{n}^{y}(\theta, x)\right)^{T}$, $\phi^{s}(\theta, x)=\left(\phi_{1}^{s}(\theta, x), \phi_{2}^{s}(\theta, x), \ldots, \phi_{n}^{s}(\theta, x)\right)^{T} \in \mathscr{C}([-\bar{\tau}, 0) \times$ $\left.\Omega, \mathbb{R}^{n}\right)$, and $\mathscr{C}\left([-\bar{\tau}, 0) \times \Omega, \mathbb{R}^{n}\right)$ is the Banach space of continuous functions which maps $[-\bar{\tau}, 0) \times \Omega$ into $\mathbb{R}^{n}$ with the topology of uniform converge and $p$-norm ( $p$ is a positive integer) defined by

$$
\begin{aligned}
\left\|\phi^{y}\right\|_{p} & =\left(\int_{\Omega} \sum_{i=1}^{n} \sup _{-\bar{\tau} \leq \theta \leq 0}\left|\phi_{i}^{y}(\theta, x)\right|^{p} \mathrm{~d} x\right)^{1 / p}, \\
\left\|\phi^{s}\right\|_{p} & =\left(\int_{\Omega} \sum_{i=1}^{n} \sup _{-\bar{\tau} \leq \theta \leq 0}\left|\phi_{i}^{s}(\theta, x)\right|^{p} \mathrm{~d} x\right)^{1 / p} .
\end{aligned}
$$

In order to observe the exponential synchronization behavior of system (5), the response system with stochastic perturbation is designed as

$$
\begin{aligned}
& \text { STM: } \mathrm{d} z_{i}(t, x)=\frac{1}{\varepsilon}\left[D_{i} \Delta z_{i}(t, x)-c_{i} z_{i}\left(t-\delta_{i}(t), x\right)\right. \\
& +\sum_{j=1}^{n} a_{i j} f_{j}\left(z_{j}(t, x)\right)+\sum_{j=1}^{n} b_{i j} f_{j}\left(z_{j}\left(t-\tau_{i j}(t), x\right)\right) \\
& +\sum_{j=1}^{n} d_{i j} \int_{t-\tau_{i j}^{*}(t)}^{t} f_{j}\left(z_{j}(s, x)\right) \mathrm{d} s+H_{i} h_{i}(t, x)+u_{i}(t, \\
& x)] \mathrm{d} t+\sum_{j=1}^{n} \sigma_{i j}\left(e_{j}(t, x), e_{j}\left(t-\delta_{i}(t), x\right),\right. \\
& \left.\quad e_{j}\left(t-\tau_{i j}(t), x\right), e_{j}\left(t-\tau_{i j}^{*}(t), x\right)\right) \mathrm{d} \omega_{j}(t), \\
& \text { LTM: } \frac{\partial h_{i}(t, x)}{\partial t}=-K_{i} h_{i}(t, x)+f_{i}\left(z_{i}(t, x)\right),
\end{aligned}
$$

where $z(t, x)=\left(z_{1}(t, x), z_{2}(t, x), \ldots, z_{n}(t, x)\right)^{T}$ and $h(t, x)=$ $\left(h_{1}(t, x), h_{2}(t, x), \ldots, h_{n}(t, x)\right)^{T}$ denote the state of the response system; $e(t, x)=z(t, x)-y(t, x)$ and $R(t, x)=$ $h(t, x)-s(t, x)$ are the synchronization error system; $\sigma=$ $\left(\sigma_{i j}\right)_{n \times n}$ is the noise intensity matrix and the stochastic disturbance $\omega(t)=\left[\omega_{1}(t), \omega_{2}(t), \ldots, \omega_{n}(t)\right]^{T} \in \mathbb{R}^{n}$ is a Brownian motion defined on $(\Omega, \mathscr{F}, \mathscr{P}$ ) (where $\Omega$ is the sample, $\mathscr{F}$ is the $\sigma$-algebra of subsets of the sample space, and $\mathscr{P}$ is the probability measure on $\mathscr{F}$ ), and

$$
\begin{array}{r}
\mathbf{E}\{\mathrm{d} \omega(t)\}=0, \\
\mathbf{E}\left\{\mathrm{d} \omega^{2}(t)\right\}=0,
\end{array}
$$

where $\mathbf{E}\{\cdot\}$ is the mathematical expectation operator with respect to the given probability measure $\mathscr{P} ; u(t, x)=$ $\left(u_{1}(t, x), u_{2}(t, x), \ldots, u_{n}(t, x)\right)^{T}$ is a feedback controller of the following form:

$$
u(t, x)=\epsilon e(t, x)
$$

The feedback strength $\epsilon=\operatorname{diag}\left(\epsilon_{1}, \epsilon_{2}, \ldots, \epsilon_{n}\right)$ is updated by the following law:

$$
\frac{\partial \epsilon_{i}}{\partial t}=-v_{i}\left|e_{i}(t, x)\right|^{p} e^{\mu t},
$$

where $\mu>0$ and $\nu_{i}>0(i=1,2, \ldots, n)$ are arbitrary positive constants.

The boundary condition and initial condition for response system (9) are given in the following forms:

$$
\begin{aligned}
\frac{\partial z_{i}(t, x)}{\partial \mathbf{n}} & :=\left(\frac{\partial z_{i}(t, x)}{\partial x_{1}}, \frac{\partial z_{i}(t, x)}{\partial x_{2}}, \ldots, \frac{\partial z_{i}(t, x)}{\partial x_{m}}\right)^{T} \\
& =\mathbf{0}, \quad(t, x) \in[-\bar{\tau},+\infty) \times \partial \Omega,
\end{aligned}
$$




$$
\begin{aligned}
\frac{\partial h_{i}(t, x)}{\partial \mathbf{n}} & :=\left(\frac{\partial h_{i}(t, x)}{\partial x_{1}}, \frac{\partial h_{i}(t, x)}{\partial x_{2}}, \ldots, \frac{\partial h_{i}(t, x)}{\partial x_{m}}\right)^{T} \\
& =\mathbf{0}, \quad(t, x) \in[-\bar{\tau},+\infty) \times \partial \Omega \\
z(\theta, x) & =\psi^{z}(\theta, x), \\
h(\theta, x) & =\psi^{h}(\theta, x),
\end{aligned}
$$

$$
(\theta, x) \in[-\bar{\tau}, 0] \times \Omega,
$$

where $\psi^{z}(\theta, x)=\left(\psi_{1}^{z}(\theta, x), \psi_{2}^{z}(\theta, x), \ldots, \psi_{n}^{z}(\theta, x)\right)^{T}$ and $\psi^{h}(\theta, x)=\left(\psi_{1}^{h}(\theta, x), \psi_{2}^{h}(\theta, x), \ldots, \psi_{n}^{h}(\theta, x)\right)^{T} \in \mathscr{C}([-\bar{\tau}, 0) \times$ $\left.\Omega, \mathbb{R}^{n}\right)$.

Subtracting (5) from (9) yields the error system as follows:

$$
\begin{aligned}
& \text { STM: } \mathrm{d} e_{i}(t, x)=\frac{1}{\varepsilon}\left[D_{i} \Delta e_{i}(t, x)-c_{i} e_{i}\left(t-\delta_{i}(t), x\right)\right. \\
& +\sum_{j=1}^{n} a_{i j} f_{j}^{*}\left(e_{j}(t, x)\right)+\sum_{j=1}^{n} b_{i j} f_{j}^{*}\left(e_{j}\left(t-\tau_{i j}(t), x\right)\right) \\
& +\sum_{j=1}^{n} d_{i j} \int_{t-\tau_{i j}^{*}(t)}^{t} f_{j}^{*}\left(e_{j}(s, x)\right) \mathrm{d} s+H_{i} R_{i}(t, x) \\
& \left.+u_{i}(t, x)\right] \mathrm{d} t+\sum_{j=1}^{n} \sigma_{i j}\left(e_{j}(t, x), e_{j}\left(t-\delta_{i}(t), x\right),\right. \\
& \left.\quad e_{j}\left(t-\tau_{i j}(t), x\right), e_{j}\left(t-\tau_{i j}^{*}(t), x\right)\right) \mathrm{d} \omega_{j}(t), \\
& \operatorname{LTM} \frac{\partial R_{i}(t, x)}{\partial t}=-K_{i} R_{i}(t, x)+f_{i}^{*}\left(e_{i}(t, x)\right),
\end{aligned}
$$

where $f_{j}^{*}\left(e_{j}(\cdot, x)\right)=f_{j}\left(z_{j}(\cdot, x)\right)-f_{j}\left(y_{j}(\cdot, x)\right)$.

In this paper, we give the following hypotheses.

$\left(\mathrm{H}_{1}\right)$ There exists a positive constant $L_{i}$ such that the neuron activation function $f_{i}$ satisfies the following conditions:

$$
\left|f_{i}\left(\widehat{v}_{i}\right)-f_{i}\left(\check{v}_{i}\right)\right| \leq L_{i}\left|\widehat{v}_{i}-\check{v}_{i}\right|,
$$

where $\widehat{v}_{i}, \check{v}_{i} \in \mathbb{R}, i=1,2, \ldots, n$.

$\left(\mathrm{H}_{2}\right)$ There exists a positive constant $\eta_{i j}$ such that

$$
\begin{aligned}
& \left|\sigma_{i j}\left(\widetilde{v}_{1}, \widehat{v}_{1}, \check{v}_{1}, \breve{v}_{1}\right)-\sigma_{i j}\left(\widetilde{v}_{2}, \widehat{v}_{2}, \check{v}_{2}, \breve{v}_{2}\right)\right|^{2} \\
& \quad \leq \eta_{i j}\left(\left|\widetilde{v}_{1}-\widetilde{v}_{2}\right|^{2}+\left|\widehat{v}_{1}-\widehat{v}_{2}\right|^{2}+\left|\check{v}_{1}-\check{v}_{2}\right|^{2}\right. \\
& \left.\quad+\left|\breve{v}_{1}-\breve{v}_{2}\right|^{2}\right),
\end{aligned}
$$

for all $\widetilde{v}_{1}, \widetilde{v}_{2}, \widehat{v}_{1}, \widehat{v}_{2}, \check{v}_{1}, \check{v}_{2}, \breve{v}_{1}, \breve{v}_{2} \in \mathbb{R}$, and $\sigma_{i j}(0,0,0,0)=0$, $i, j=1,2, \ldots, n$.

$\left(\mathrm{H}_{3}\right)$ There exist positive constants $\rho^{\prime}$ and $\rho^{\prime \prime}$ such that $\dot{\delta}_{i}(t) \leq \rho^{\prime}<1$ or $\dot{\delta}_{i}(t) \geq \rho^{\prime \prime}>1$ for all $t, i=1,2, \ldots, n$.

$\left(\mathrm{H}_{4}\right)$ There exist positive constants $\varrho^{\prime}$ and $\varrho^{\prime \prime}$ such that $\dot{\tau}_{i j}(t) \leq \varrho^{\prime}<1$ or $\dot{\tau}_{i j}(t) \geq \varrho^{\prime \prime}>1$ for all $t, i, j=1,2, \ldots, n$.

$\left(\mathrm{H}_{5}\right)$ There exist positive constants $\varrho^{* \prime}$ and $\varrho^{* \prime \prime}$ such that $\dot{\tau}_{i j}^{*}(t) \leq \varrho^{* \prime}<1$ or $\dot{\tau}_{i j}(t) \geq \varrho^{* \prime \prime}>1$ for all $t, i, j=1,2, \ldots, n$.
The paper is organized as follows. In the next section, we introduce some definitions and state several lemmas which will be essential to our proofs. In Section 3, by constructing a suitable Lyapunov functional, some new criteria are obtained to ensure the exponential synchronization of systems (5) and (9) under the adaptive feedback controller (11) and (12). Numerical simulations are carried out in Section 4 to illustrate the feasibility of the main theoretical results. A brief conclusion is given in Section 5.

\section{Preliminary}

In this section, we introduce some notations and lemmas which will be useful in the next section.

Definition 1. The noise-perturbed response system (9) and the drive system (5) can be exponentially synchronized under the adaptive controller (11) and (12) based on $p$-norm, if there exist constants $\gamma, \gamma^{*}>0$ and $M, M^{*} \geq 1$ such that

$$
\begin{aligned}
& \mathbf{E}\left\{\|z(t, x)-y(t, x)\|_{p}\right\}+\mathbf{E}\left\{\|h(t, x)-s(t, x)\|_{p}\right\} \\
& \leq \operatorname{ME}\left\{\left\|\psi^{z}-\phi^{y}\right\|_{p}\right\} e^{-\gamma t} \\
& +M^{*} \mathbf{E}\left\{\left\|\psi^{h}-\phi^{s}\right\|_{p}\right\} e^{-\gamma^{*} t}, \\
& \quad(t, x) \in[0,+\infty) \times \Omega,
\end{aligned}
$$

where $z(t, x), h(t, x)$ and $y(t, x), s(t, x)$ are solutions of systems (9) and (5) with differential initial functions (14) and (7), respectively, and

$$
\begin{aligned}
& \|z(t, x)-y(t, x)\|_{p} \\
& =\left(\int_{\Omega} \sum_{i=1}^{n}\left|z_{i}(t, x)-y_{i}(t, x)\right|^{p} \mathrm{~d} x\right)^{1 / p}, \\
& \|h(t, x)-s(t, x)\|_{p} \\
& =\left(\int_{\Omega} \sum_{i=1}^{n}\left|h_{i}(t, x)-s_{i}(t, x)\right|^{p} \mathrm{~d} x\right)^{1 / p} .
\end{aligned}
$$

Lemma 2 (Wang [26], Itô's formula). Let $x(t)(t \geq 0)$ be Itô processes, and

$$
\mathrm{d} x(t)=f(t) \mathrm{d} t+g(t) \mathrm{d} B_{t},
$$

where $f \in \mathscr{L}^{1}\left(R^{+}, R^{n}\right)$ ( $\mathscr{L}^{1}$ is the space of absolutely integrable function) and $g \in \mathscr{L}^{2}\left(R^{+}, R^{n \times m}\right)$ ( $\mathscr{L}^{2}$ is the space of square integrable function). If $V(x, t) \in C^{2,1}\left(R^{n} \times R^{+} ; R\right),\left(C^{2,1}\left(R^{n} \times\right.\right.$ $\left.R^{+} ; R\right)$ is the family of all nonnegative functions on $R^{+} \times R^{n}$ which are continuously twice differentiable in $x$ and once differentiable in $t)$, then $V(x(t), t)$ are still Itô processes, and

$$
\begin{aligned}
\mathrm{d} V & (x(t), t)=\left[V_{t}(x(t), t)+V_{x}(x(t), t) f(t)\right. \\
& \left.+\frac{1}{2} \operatorname{tr}\left(g^{T}(t) V_{x x}(x(t), t) g(t)\right)\right] \mathrm{d} t \\
& +V_{x}(x(t), t) g(t) \mathrm{d} B_{t},
\end{aligned}
$$


where

$$
\begin{aligned}
V_{t}(x(t), t) & =\frac{\partial V(x(t), t)}{\partial t}, \\
V_{x}(x(t), t) & =\left(\frac{\partial V(x(t), t)}{\partial x_{1}}, \ldots, \frac{\partial V(x(t), t)}{\partial x_{n}}\right), \\
V_{x x}(x(t), t) & =\left(\frac{\partial^{2} V(x(t), t)}{\partial e_{i} \partial e_{j}}\right)_{n \times n} .
\end{aligned}
$$

Lemma 3 (Mei et al. [27]). Let $p \geq 2$ and let $a, b, h>0$. Then

$$
\begin{aligned}
a^{p-1} b & \leq \frac{(p-1) h a^{p}}{p}+\frac{b^{p}}{p h^{p-1}}, \\
a^{p-2} b^{2} & \leq \frac{(p-2) h a^{p}}{p}+\frac{2 b^{p}}{p h^{(p-2) / 2}} .
\end{aligned}
$$

Lemma 4 (Mao [28]). Let $F(x), G(x):[a, b] \rightarrow \mathbb{R}$ be continuous functions. Suppose that positive constants $p$ and $q$ satisfy

$$
\frac{1}{p}+\frac{1}{q}=1
$$

Then

$$
\begin{aligned}
& \int_{a}^{b}|F(x) G(x)| \mathrm{d} x \\
& \quad \leq\left[\int_{a}^{b}|F(x)|^{p} \mathrm{~d} x\right]^{1 / p}\left[\int_{a}^{b}|G(x)|^{q} \mathrm{~d} x\right]^{1 / q} .
\end{aligned}
$$

Lemma 5 (Gu et al. [29]). Suppose that $\Omega$ is a bound domain of $R^{m}$ with a smooth boundary $\partial \Omega . u(x), v(x)$ are real-valued functions belonging to $\mathscr{C}^{2}(\Omega \cup \partial \Omega)$. Then

$$
\begin{aligned}
\int_{\Omega} u(x) \Delta v(x) \mathrm{d} x= & \int_{\partial \Omega} u(x) \frac{\partial v(x)}{\partial \mathbf{n}} \mathrm{d} x \\
& -\int_{\Omega}(\nabla u(x))^{T} \nabla v(x) \mathrm{d} x,
\end{aligned}
$$

where $\nabla=\left(\partial / \partial x_{1}, \partial / \partial x_{2}, \ldots, \partial / \partial x_{m}\right)^{T}$ is the gradient operator.

Lemma 6. Let $p \geq 2$ be a positive integer and let $\Omega$ be a bound domain of $R^{l^{*}}$ with a smooth boundary $\partial \Omega . \varphi(x) \in \mathscr{C}^{1}(\Omega)$ is a real-valued function and $\left.(\partial \varphi(x) / \partial \mathbf{n})\right|_{\partial \Omega}=\mathbf{0}$. Then

$$
\int_{\Omega}|\varphi(x)|^{p} \mathrm{~d} x \leq \frac{p-1}{\lambda_{1}} \int_{\Omega}|\varphi(x)|^{p-2}|\nabla \varphi(x)|^{2} \mathrm{~d} x,
$$

where $\lambda_{1}$ is the smallest positive eigenvalue of the Neumann boundary problem:

$$
-\Delta \vartheta(x)=\lambda \vartheta(x), \quad x \in \Omega,
$$

$$
\frac{\partial \vartheta(x)}{\partial \mathbf{n}}=0, \quad x \in \partial \Omega
$$

The proof of Lemma 6 is attached in Appendix.

Remark 7. If $p=2$, the integral inequality (28) is the Poincare integral inequality in [30]. The smallest eigenvalue $\lambda_{1}$ of the Neumann boundary problem (29) is determined by the boundary of $\Omega$ [30]. If $\Omega=\left\{x=\left(x_{1}, x_{2}, \ldots, x_{l^{*}}\right)^{T} \mid m_{k}^{-} \leq\right.$ $\left.x_{k} \leq m_{k}^{+}, k=1,2, \ldots, l^{*}\right\} \subset R^{l^{*}}$, then

$$
\begin{gathered}
\lambda_{1}=\min \left\{\left(\frac{\pi}{m_{1}^{+}-m_{1}^{-}}\right)^{2},\left(\frac{\pi}{m_{2}^{+}-m_{2}^{-}}\right)^{2}, \ldots,\right. \\
\left.\left(\frac{\pi}{m_{l^{*}}^{+}-m_{l^{*}}^{-}}\right)^{2}\right\} .
\end{gathered}
$$

\section{Exponential Synchronization Criterion}

In this section, the exponential synchronization criterion of the drive system (5) and the response system (9) is obtained under the adaptive feedback controller (11) and (12). For convenience, the following denotations are introduced.

Denote

$$
\begin{aligned}
& \alpha_{i}=\frac{1}{\kappa|1-\rho| e^{\mu \delta}}\left[\frac{1}{\varepsilon} \frac{1}{\xi_{i}^{* * p-1}}+(p-1) \sum_{j=1}^{n} \frac{\eta_{j i}}{\varsigma_{i}^{*((p-2) / 2)}}\right], \\
& \beta_{i j}=\frac{1}{e^{\mu \tau} \kappa|1-\varrho|}\left[\frac{(p-1) \eta_{j i}}{\varsigma_{i}^{* *((p-2) / 2)}}+\frac{1}{\varepsilon} \frac{\left|b_{j i}\right| L_{i}}{\omega_{i}^{* p-1}}\right], \\
& \gamma_{i j}=\frac{(p-1) \eta_{j i}}{e^{\mu \tau^{*}} \kappa\left|1-\varrho^{*}\right| \varsigma_{i}^{* * *((p-2) / 2)}}, \\
& \alpha_{i}^{*}=\alpha_{i}-\kappa \alpha_{i} \operatorname{sgn}(1-\rho) \text {, } \\
& \beta_{i j}^{*}=\beta_{i j}-\kappa \beta_{i j} \operatorname{sgn}(1-\varrho) \text {, } \\
& \gamma_{i j}^{*}=\gamma_{i j}-\kappa \gamma_{i j} \operatorname{sgn}\left(1-\varrho^{*}\right), \\
& \gamma_{i j}^{* *}=\frac{1}{\varepsilon} \frac{\left(\tau^{*}\right)^{p-1} L_{i}\left|d_{j i}\right|}{\omega_{i}^{* * p-1}}, \\
& l_{i}=\frac{\varepsilon}{p}\left[-\frac{1}{\varepsilon} p \lambda_{1} D_{i}+\mu+\alpha_{i} e^{\mu \delta}+\frac{1}{\varepsilon} c_{i}(p-1) \xi_{i}^{* *}\right. \\
& +\frac{L_{i}}{\xi_{i}^{p-1}}+\frac{1}{\varepsilon}\left|H_{i}\right|(p-1) \xi_{i}^{*}+e^{\mu \tau} \sum_{j=1}^{n} \beta_{i j}+e^{\mu \tau^{*}} \sum_{j=1}^{n} \gamma_{i j} \\
& +\tau^{*} e^{\mu \tau^{*}} \sum_{j=1}^{n} \gamma_{i j}^{* *}+\frac{1}{\varepsilon} \sum_{j=1}^{n} \frac{1}{\omega_{i}^{p-1}}\left|a_{j i}\right| L_{i}
\end{aligned}
$$




$$
\begin{aligned}
& +\frac{1}{\varepsilon}(p-1) \sum_{j=1}^{n}\left|b_{i j}\right| L_{j} \omega_{j}^{*} \\
& +\frac{1}{\varepsilon}(p-1) \sum_{j=1}^{n}\left|d_{i j}\right| L_{j} \omega_{j}^{* *} \\
& +\frac{1}{\varepsilon}(p-1) \sum_{j=1}^{n}\left|a_{i j}\right| L_{j} \omega_{j}+\frac{(p-1)(p-2)}{2} \sum_{j=1}^{n} \eta_{i j} \varsigma_{j} \\
& +\frac{(p-1)(p-2)}{2} \sum_{j=1}^{n} \eta_{i j} \varsigma_{j}^{*} \\
& +\frac{(p-1)(p-2)}{2} \sum_{j=1}^{n} \eta_{i j} \varsigma_{j}^{* *} \\
& \left.+\frac{(p-1)(p-2)}{2} \sum_{j=1}^{n} \eta_{i j} \varsigma_{j}^{* * *}+(p-1) \sum_{j=1}^{n} \frac{\eta_{j i}^{(p-2) / 2}}{\varsigma_{i}^{(}}\right],
\end{aligned}
$$

where $0<\kappa<1,|1-\rho|=\max \left\{\left|1-\rho^{\prime}\right|,\left|1-\rho^{\prime \prime}\right|\right\},|1-\varrho|=$ $\max \left\{\left|1-\varrho^{\prime}\right|,\left|1-\varrho^{\prime \prime}\right|\right\},\left|1-\varrho^{*}\right|=\max \left\{\left|1-\varrho^{* \prime}\right|,\left|1-\varrho^{* \prime \prime}\right|\right\}$, and $\xi_{i}, \xi_{i}^{*}, \xi_{i}^{* *}, \varsigma_{i}, \varsigma_{i}^{*}, \varsigma_{i}^{* *}, \varsigma_{i}^{* * *}, \omega_{i}, \omega_{i}^{*}$, and $\omega_{i}^{* *}$ are nonnegative real numbers, respectively.

Theorem 8. Under assumptions $\left(\mathrm{H}_{1}\right)-\left(\mathrm{H}_{5}\right)$, the nonlinear couple neural networks (9) and (5) can be exponentially synchronized under the adaptive feedback controller (11) and (12) based on $p$-norm, if the following condition is also satisfied.

$\left(\mathrm{H}_{6}\right) \mu-p K_{i}+L_{i}(p-1) \xi_{i}+(1 / \varepsilon)\left(\left|H_{i}\right| / \xi_{i}^{*}{ }^{-1}\right) \leq 0$.
Proof. Define

$$
\begin{aligned}
& V(t)=\int_{\Omega} \sum_{i=1}^{n}\left[V_{i}(t, x)+\alpha_{i} e^{\mu \delta} \int_{t-\delta_{i}(t)}^{t} V_{i}(s, x) \mathrm{d} s\right. \\
& +\alpha_{i}^{*} e^{\mu \delta} \int_{t-\delta}^{t-\delta_{i}(t)} V_{i}(s, x) \mathrm{d} s \\
& +e^{\mu \tau} \sum_{j=1}^{n} \beta_{i j} \int_{t-\tau_{i j}(t)}^{t} V_{i}(s, x) \mathrm{d} s \\
& +e^{\mu \tau} \sum_{j=1}^{n} \beta_{i j}^{*} \int_{t-\tau}^{t-\tau_{i j}(t)} V_{i}(s, x) \mathrm{d} s \\
& +e^{\mu \tau^{*}} \sum_{j=1}^{n} \gamma_{i j} \int_{t-\tau_{i j}^{*}(t)}^{t} V_{i}(s, x) \mathrm{d} s \\
& \left.+e^{\mu t}\left|R_{i}(t, x)\right|^{p}+\frac{p}{2 \varepsilon \nu_{i}}\left(\epsilon_{i}+l_{i}\right)^{2}\right] \mathrm{d} x \\
& +e^{\mu \tau^{*}} \sum_{j=1}^{n} \gamma_{i j}^{*} \int_{t-\tau^{*}}^{t-\tau_{i j}^{*}(t)} V_{i}(s, x) \mathrm{d} s \\
& +e^{\mu \tau^{*}} \sum_{j=1}^{n} \gamma_{i j}^{* *} \int_{-\tau^{*}}^{0} \int_{t+s}^{t} V_{i}(\eta, x) \mathrm{d} \eta \mathrm{d} s
\end{aligned}
$$

where $V_{i}(t, x)=e^{\mu t}\left|e_{i}(t, x)\right|^{p}$.

By (10), Itô's differential formula, and Dini derivation, it can be deduced that

$$
\begin{aligned}
& D^{+} \mathbf{E}\{V(t)\}=\mathbf{E}\left\{\int _ { \Omega } \sum _ { i = 1 } ^ { n } \left\{\mu V_{i}(t, x)+\alpha_{i} e^{\mu \delta}\left[V_{i}(t, x)-V_{i}\left(t-\delta_{i}(t), x\right)\left(1-\dot{\delta}_{i}(t)\right)\right]\right.\right. \\
& +\alpha_{i}^{*} e^{\mu \delta}\left[V_{i}\left(t-\delta_{i}(t), x\right)\left(1-\dot{\delta}_{i}(t)\right)-V_{i}(t-\delta, x)\right] \\
& +e^{\mu \tau} \sum_{j=1}^{n} \beta_{i j}\left[V_{i}(t, x)-V_{i}\left(t-\tau_{i j}(t), x\right)\left(1-\dot{\tau}_{i j}(t)\right)\right] \\
& +e^{\mu \tau} \sum_{j=1}^{n} \beta_{i j}^{*}\left[V_{i}\left(t-\tau_{i j}(t), x\right)\left(1-\dot{\tau}_{i j}(t)\right)-V_{i}(t-\tau, x)\right] \\
& +e^{\mu \tau^{*}} \sum_{j=1}^{n} \gamma_{i j}\left[V_{i}(t, x)-V_{i}\left(t-\tau_{i j}^{*}(t), x\right)\left(1-\dot{\tau}_{i j}^{*}(t)\right)\right] \\
& +e^{\mu \tau^{*}} \sum_{j=1}^{n} \gamma_{i j}^{*}\left[V_{i}\left(t-\tau_{i j}^{*}(t), x\right)\left(1-\dot{\tau}_{i j}^{*}(t)\right)-V_{i}\left(t-\tau^{*}, x\right)\right] \\
& +e^{\mu \tau^{*}} \sum_{j=1}^{n} \gamma_{i j}^{* *} \int_{-\tau^{*}}^{0}\left[V_{i}(t, x)-V_{i}(t+s, x)\right] \mathrm{d} s+\mu e^{\mu t}\left|R_{i}(t, x)\right|^{p} \\
& +p e^{\mu t}\left|R_{i}(t, x)\right|^{p-1}\left[-K_{i} R_{i}(t, x)+f_{i}^{*}\left(e_{i}(t, x)\right)\right]
\end{aligned}
$$


Mathematical Problems in Engineering

7

$$
\begin{aligned}
& -\frac{p}{\varepsilon}\left(\epsilon_{i}+l_{i}\right)\left|e_{i}(t, x)\right|^{p} e^{\mu t} \\
& +\frac{1}{\varepsilon} p e^{\mu t}\left|e_{i}(t, x)\right|^{p-1}\left[D_{i} \Delta e_{i}(t, x)-c_{i} e_{i}\left(t-\delta_{i}(t), x\right)\right. \\
& +\sum_{j=1}^{n} a_{i j} f_{j}^{*}\left(e_{j}(t, x)\right) \\
& +\sum_{j=1}^{n} b_{i j} f_{j}^{*}\left(e_{j}\left(t-\tau_{i j}(t), x\right)\right) \\
& \left.+H_{i} R_{i}(t, x)+\epsilon_{i} e_{i}(t, x)\right] \\
& +\frac{p(p-1) e^{\mu t}\left|e_{i}(t, x)\right|^{p-2}}{2} \\
& \cdot \sum_{j=1}^{n} \sigma_{i j}^{2}\left(e_{j}(t, x), e_{j}\left(t-\delta_{i}(t), x\right)\right. \\
& \left.\left.\left.e_{j}\left(t-\tau_{i j}(t), x\right), e_{j}\left(t-\tau_{i j}^{*}(t), x\right)\right)\right\} \mathrm{d} x\right\} \\
& \leq \mathbf{E}\left\{\int _ { \Omega } \sum _ { i = 1 } ^ { n } \left\{\mu V_{i}(t, x)+\alpha_{i} e^{\mu \delta} V_{i}(t, x)\right.\right. \\
& -\kappa \alpha_{i}|1-\rho| e^{\mu \delta} V_{i}\left(t-\delta_{i}(t), x\right) \\
& +e^{\mu \tau} \sum_{j=1}^{n} \beta_{i j} V_{i}(t, x)-e^{\mu \tau} \sum_{j=1}^{n} \kappa \beta_{i j}|1-\varrho| V_{i}\left(t-\tau_{i j}(t), x\right) \\
& +e^{\mu \tau^{*}} \sum_{j=1}^{n} \gamma_{i j} V_{i}(t, x)-e^{\mu \tau^{*}} \sum_{j=1}^{n} \kappa \gamma_{i j}\left|1-\varrho^{*}\right| V_{i}\left(t-\tau_{i j}^{*}(t), x\right) \\
& +\tau^{*} e^{\mu \tau^{*}} \sum_{j=1}^{n} \gamma_{i j}^{* *} V_{i}(t, x)-e^{\mu \tau^{*}} \sum_{j=1}^{n} \gamma_{i j}^{* *} \int_{t-\tau^{*}}^{t} V_{i}(s, x) \mathrm{d} s \\
& +\left(\mu-p K_{i}\right) e^{\mu t}\left|R_{i}(t, x)\right|^{p}+p e^{\mu t}\left|R_{i}(t, x)\right|^{p-1} L_{i}\left|e_{i}(t, x)\right| \\
& -\frac{p}{\varepsilon}\left(\epsilon_{i}+l_{i}\right)\left|e_{i}(t, x)\right|^{p} e^{\mu t}+\frac{1}{\varepsilon} p e^{\mu t}\left|e_{i}(t, x)\right|^{p-1}\left[D_{i} \Delta\left|e_{i}(t, x)\right|+c_{i}\left|e_{i}\left(t-\delta_{i}(t), x\right)\right|\right. \\
& +\sum_{j=1}^{n}\left|a_{i j}\right| L_{j}\left|e_{j}(t, x)\right|+\sum_{j=1}^{n}\left|b_{i j}\right| L_{j}\left|e_{j}\left(t-\tau_{i j}(t), x\right)\right| \\
& +\sum_{j=1}^{n}\left|d_{i j}\right| \int_{t-\tau_{i j}^{*}(t)}^{t} L_{j}\left|e_{j}(s, x)\right| \mathrm{d} s \\
& \left.+\left|H_{i}\right|\left|R_{i}(t, x)\right|+\epsilon_{i}\left|e_{i}(t, x)\right|\right]+\frac{p(p-1) e^{\mu t}\left|e_{i}(t, x)\right|^{p-2}}{2} \\
& \sum_{j=1}^{n} \eta_{i j}\left(\left|e_{j}(t, x)\right|^{2}+\left|e_{j}\left(t-\delta_{i}(t), x\right)\right|^{2}\right. \\
& \left.\left.\left.+\left|e_{j}\left(t-\tau_{i j}(t), x\right)\right|^{2}+\left|e_{j}\left(t-\tau_{i j}^{*}(t), x\right)\right|^{2}\right)\right\} \mathrm{~d} x\right\} .
\end{aligned}
$$


From the boundary conditions (6) and (13) and Lemma 6, we get

$$
\begin{aligned}
& p \int_{\Omega}\left|e_{i}(t, x)\right|^{p-1} D_{i} \Delta\left|e_{i}(t, x)\right| \mathrm{d} x \\
& =p\left(\int_{\partial \Omega}\left|e_{i}(t, x)\right|^{p-1}\right. \\
& \cdot D_{i} \sum_{k=1}^{m} \frac{\partial\left|e_{i}(t, x)\right|}{\partial x_{k}} \cos \left(x_{k}, n\right) \mathrm{d} s \\
& \left.-\int_{\Omega} \sum_{k=1}^{m} D_{i} \frac{\partial\left|e_{i}(t, x)\right|}{\partial x_{k}} \cdot \frac{\partial\left|e_{i}(t, x)\right|^{p-1}}{\partial x_{k}} \mathrm{~d} x\right) \\
& =-p(p-1) D_{i} \int_{\Omega}\left|e_{i}(t, x)\right|^{p-2} \sum_{k=1}^{m} \frac{\partial\left|e_{i}(t, x)\right|}{\partial x_{k}} \\
& \quad \cdot \frac{\partial\left|e_{i}(t, x)\right|}{\partial x_{k}} \mathrm{~d} x=-p(p-1) \\
& \left.\cdot D_{i} \int_{\Omega}\left|e_{i}(t, x)\right|^{p-2}|\nabla| e_{i}(t, x)\right|^{2} \mathrm{~d} x \\
& \quad \leq-p \lambda_{1} D_{i} \int_{\Omega}\left|e_{i}(t, x)\right|^{p} \mathrm{~d} x .
\end{aligned}
$$

By Lemma 4, we obtain

$$
\begin{gathered}
\int_{t-\tau^{*}}^{t} V_{i}(s, x) \mathrm{d} s \geq e^{\mu t} e^{-\mu \tau^{*}} \int_{t-\tau_{i j}^{*}(t)}^{t}\left|e_{i}(s, x)\right|^{p} \mathrm{~d} s \\
\geq e^{\mu t} e^{-\mu \tau^{\star}} \frac{\left(\int_{t-\tau_{i j}^{*}(t)}^{t}\left|e_{i}(s, x)\right| \mathrm{d} s\right)^{p}}{\left(\tau_{i j}^{*}(t)\right)^{p / q}} \\
\geq e^{\mu t} e^{-\mu \tau^{\star}}\left(\tau^{*}\right)^{1-p}\left(\int_{t-\tau_{i j}^{*}(t)}^{t}\left|e_{i}(s, x)\right| \mathrm{d} s\right)^{p} .
\end{gathered}
$$

It follows from (23) that

$$
\begin{aligned}
& p\left|R_{i}(t, x)\right|^{p-1}\left|e_{i}(t, x)\right| \\
& \leq(p-1) \xi_{i}\left|R_{i}(t, x)\right|^{p}+\frac{\left|e_{i}(t, x)\right|^{p}}{\xi_{i}^{p-1}}, \\
& p\left|e_{i}(t, x)\right|^{p-1}\left|R_{i}(t, x)\right| \\
& \quad \leq(p-1) \xi_{i}^{*}\left|e_{i}(t, x)\right|^{p}+\frac{\left|R_{i}(t, x)\right|^{p}}{\xi_{i}^{* p-1}},
\end{aligned}
$$

$p\left|e_{i}(t, x)\right|^{p-1}\left|e_{i}\left(t-\delta_{i}(t), x\right)\right|$

$$
\leq(p-1) \xi_{i}^{* *}\left|e_{i}(t, x)\right|^{p}+\frac{\left|e_{i}\left(t-\delta_{i}(t), x\right)\right|^{p}}{\xi_{i}^{* * p-1}},
$$

$$
\begin{gathered}
p\left|e_{i}(t, x)\right|^{p-1} \sum_{j=1}^{n}\left|a_{i j}\right| L_{j}\left|e_{j}(t, x)\right| \\
\leq(p-1)\left|e_{i}(t, x)\right|^{p} \sum_{j=1}^{n}\left|a_{i j}\right| L_{j} \Theta_{j} \\
+\sum_{j=1}^{n} \frac{L_{j}\left|a_{i j}\right|\left|e_{j}(t, x)\right|^{p}}{\omega_{j}^{p-1}}
\end{gathered}
$$

$$
\begin{gathered}
p\left|e_{i}(t, x)\right|^{p-1} \sum_{j=1}^{n}\left|b_{i j}\right| L_{j}\left|e_{j}\left(t-\tau_{i j}(t), x\right)\right| \\
\leq(p-1)\left|e_{i}(t, x)\right|^{p} \sum_{j=1}^{n}\left|b_{i j}\right| L_{j} \varpi_{j}^{*} \\
+\sum_{j=1}^{n} \frac{L_{j}\left|b_{i j}\right|\left|e_{j}\left(t-\tau_{i j}(t), x\right)\right|^{p}}{\omega_{j}^{* p-1}}
\end{gathered}
$$$$
p\left|e_{i}(t, x)\right|^{p-1} \sum_{j=1}^{n}\left|d_{i j}\right| L_{j} \int_{t-\tau_{i j}^{*}(t)}^{t}\left|e_{j}(s, x)\right| \mathrm{d} s
$$

$$
\begin{aligned}
& \leq(p-1)\left|e_{i}(t, x)\right|^{p} \sum_{j=1}^{n}\left|d_{i j}\right| L_{j}{\varpi_{j}^{* *}}^{*} \\
& \quad+\sum_{j=1}^{n} \frac{\left|d_{i j}\right| L_{j}}{\omega_{j}^{* * p-1}}\left(\int_{t-\tau_{i j}^{*}(t)}^{t}\left|e_{j}(s, x)\right| \mathrm{d} s\right)^{p} .
\end{aligned}
$$

It follows from (24) that

$$
\begin{aligned}
& p\left|e_{i}(t, x)\right|^{p-2} \sum_{j=1}^{n} \eta_{i j}\left|e_{j}(t, x)\right|^{2} \\
& \quad \leq(p-2)\left|e_{i}(t, x)\right|^{p} \sum_{j=1}^{n} \eta_{i j} \varsigma_{j}+\sum_{j=1}^{n} \frac{2 \eta_{i j}\left|e_{j}(t, x)\right|^{p}}{\varsigma_{j}^{(p-2) / 2}},
\end{aligned}
$$

$p\left|e_{i}(t, x)\right|^{p-2} \sum_{j=1}^{n} \eta_{i j}\left|e_{j}\left(t-\delta_{i}(t)\right)\right|^{2}$

$$
\leq(p-2)\left|e_{i}(t, x)\right|^{p} \sum_{j=1}^{n} \eta_{i j} \varsigma_{j}^{*}
$$


Mathematical Problems in Engineering

9

$$
\begin{gathered}
+\sum_{j=1}^{n} \frac{2 \eta_{i j}\left|e_{j}\left(t-\delta_{i}(t)\right)\right|^{p}}{\varsigma_{j}^{*((p-2) / 2)}} \\
p\left|e_{i}(t, x)\right|^{p-2} \sum_{j=1}^{n} \eta_{i j}\left|e_{j}\left(t-\tau_{i j}(t)\right)\right|^{2} \\
\leq(p-2)\left|e_{i}(t, x)\right|^{p} \sum_{j=1}^{n} \eta_{i j} \varsigma_{j}^{* *} \\
+\sum_{j=1}^{n} \frac{2 \eta_{i j}\left|e_{j}\left(t-\tau_{i j}(t)\right)\right|^{p}}{\varsigma_{j}^{* *((p-2) / 2)}}
\end{gathered}
$$

$$
\begin{gathered}
p\left|e_{i}(t, x)\right|^{p-2} \sum_{j=1}^{n} \eta_{i j}\left|e_{j}\left(t-\tau_{i j}^{*}(t)\right)\right|^{2} \\
\leq(p-2)\left|e_{i}(t, x)\right|^{p} \sum_{j=1}^{n} \eta_{i j} \varsigma_{j}^{* * *} \\
+\sum_{j=1}^{n} \frac{2 \eta_{i j}\left|e_{j}\left(t-\tau_{i j}^{*}(t)\right)\right|^{p}}{\varsigma_{j}^{* * *((p-2) / 2)}} .
\end{gathered}
$$

Substituting (34)-(37) into (33), it follows from (31) and $\left(\mathrm{H}_{5}\right)$ that

$$
\begin{aligned}
& D^{+} \mathbf{E}\{V(t)\} \leq \mathbf{E}\left\{\int _ { \Omega } \sum _ { i = 1 } ^ { n } \left\{\left[\mu+\alpha_{i} e^{\mu \delta}+e^{\mu \tau} \sum_{j=1}^{n} \beta_{i j}\right.\right.\right. \\
& +e^{\mu \tau^{*}} \sum_{j=1}^{n} \gamma_{i j}+\tau^{*} e^{\mu \tau^{*}} \sum_{j=1}^{n} \gamma_{i j}^{* *} \\
& +\frac{L_{i}}{\xi_{i}^{p-1}}-\frac{p}{\varepsilon} l_{i}-\frac{1}{\varepsilon} p \lambda_{1} D_{i} \\
& +\frac{1}{\varepsilon} c_{i}(p-1) \xi_{i}^{* *}+\frac{1}{\varepsilon}(p-1) \sum_{j=1}^{n}\left|a_{i j}\right| L_{j} \varrho_{j} \\
& +\frac{1}{\varepsilon}(p-1) \sum_{j=1}^{n}\left|b_{i j}\right| L_{j} \omega_{j}^{*} \\
& +\frac{1}{\varepsilon}(p-1) \sum_{j=1}^{n}\left|d_{i j}\right| L_{j} \omega_{j}^{* *} \\
& +\frac{1}{\varepsilon}\left|H_{i}\right|(p-1) \xi_{i}^{*}+\frac{1}{\varepsilon} \sum_{j=1}^{n} \frac{1}{\omega_{i}^{p-1}}\left|a_{j i}\right| L_{i} \\
& +\frac{1}{2}(p-1)(p-2) \sum_{j=1}^{n} \eta_{i j} \varsigma_{j} \\
& +\frac{1}{2}(p-1)(p-2) \sum_{j=1}^{n} \eta_{i j} \varsigma_{j}^{*} \\
& +\frac{1}{2}(p-1)(p-2) \sum_{j=1}^{n} \eta_{i j} \varsigma_{j}^{* *} \\
& +\frac{1}{2}(p-1)(p-2) \sum_{j=1}^{n} \eta_{i j} \varsigma_{j}^{* * *} \\
& \left.+(p-1) \sum_{j=1}^{n} \frac{\eta_{j i}}{\varsigma_{i}^{(p-2) / 2}}\right] V_{i}(t, x)
\end{aligned}
$$




$$
\begin{aligned}
& +\left[\frac{1}{\varepsilon} \frac{1}{\xi_{i}^{* * p-1}}+(p-1) \sum_{j=1}^{n} \frac{\eta_{j i}}{\varsigma_{i}^{*((p-2) / 2)}}\right. \\
& \left.-\kappa \alpha_{i}|1-\rho| e^{\mu \delta}\right] V_{i}\left(t-\delta_{i}(t), x\right) \\
& +\left[(p-1) \sum_{j=1}^{n} \frac{\eta_{j i}}{\varsigma_{i}^{* *((p-2) / 2)}}+\frac{1}{\varepsilon} \sum_{j=1}^{n} \frac{\left|b_{j i}\right| L_{i}}{\omega_{i}^{* p-1}}\right. \\
& \left.-e^{\mu \tau} \sum_{j=1}^{n} \kappa \beta_{i j}|1-\varrho|\right] V_{i}\left(t-\tau_{i j}(t), x\right) \\
& +\left[(p-1) \sum_{j=1}^{n} \frac{\eta_{j i}}{\varsigma_{i}^{* * *((p-2) / 2)}}\right. \\
& \left.-e^{\mu \tau^{*}} \sum_{j=1}^{n} \kappa \gamma_{i j}\left|1-\varrho^{*}\right|\right] V_{i}\left(t-\tau_{i j}^{*}(t), x\right) \\
& +\left[\frac{1}{\varepsilon} \sum_{j=1}^{n} \frac{\left|d_{j i}\right| L_{i}}{\omega_{i}^{* * p-1}}-\sum_{j=1}^{n} \gamma_{i j}^{* *}\left(\tau^{*}\right)^{1-p}\right] \\
& \cdot e^{\mu t}\left(\int_{t-\tau_{i j}^{*}(t)}^{t}\left|e_{i}(s, x)\right| \mathrm{d} s\right)^{p} \\
& +\left(\frac{1}{\varepsilon} \frac{\left|H_{i}\right|}{\xi_{i}^{* p-1}}+\mu-p K_{i}+L_{i}(p-1) \xi_{i}\right) \\
& \left.\left.e^{\mu t}\left|R_{i}(t, x)\right|^{p}\right\} \mathrm{~d} x\right\} \\
& =\mathbf{E}\left\{\int_{\Omega} \sum_{i=1}^{n}\left\{\left(\frac{1}{\varepsilon} \frac{\left|H_{i}\right|}{\xi_{i}^{* p-1}}+\mu-p K_{i}+L_{i}(p-1) \xi_{i}\right) e^{\mu t}\left|R_{i}(t, x)\right|^{p}\right\} \mathrm{d} x\right\} \leq 0
\end{aligned}
$$

which implies that

Note that

$$
\mathbf{E}\{V(t)\} \leq \mathbf{E}\{V(0)\} .
$$

$$
\begin{gathered}
\mathbf{E}\{V(0)\} \leq \mathbf{E}\left\{\int _ { \Omega } \sum _ { i = 1 } ^ { n } \left[\left|e_{i}(0, x)\right|^{p}\right.\right. \\
+\alpha_{i} e^{\mu \delta} \int_{-\delta_{i}(0)}^{0} e^{\mu s}\left|e_{i}(s, x)\right|^{p} \mathrm{~d} s
\end{gathered}
$$


Mathematical Problems in Engineering

11

$$
\begin{aligned}
& +\alpha_{i}^{*} e^{\mu \delta} \int_{-\delta}^{-\delta_{i}(0)} e^{\mu s}\left|e_{i}(s, x)\right|^{p} \mathrm{~d} s \\
& +e^{\mu \tau} \sum_{j=1}^{n} \beta_{i j} \int_{-\tau_{i j}(0)}^{0} e^{\mu s}\left|e_{i}(s, x)\right|^{p} \mathrm{~d} s \\
& +e^{\mu \tau} \sum_{j=1}^{n} \beta_{i j}^{*} \int_{-\tau}^{-\tau_{i j}(0)} e^{\mu s}\left|e_{i}(s, x)\right|^{p} \mathrm{~d} s \\
& +e^{\mu \tau^{*}} \sum_{j=1}^{n} \gamma_{i j} \int_{-\tau_{i j}^{*}(0)}^{0} e^{\mu s}\left|e_{i}(s, x)\right|^{p} \mathrm{~d} s \\
& +e^{\mu \tau^{*}} \sum_{j=1}^{n} \gamma_{i j}^{*} \int_{-\tau^{*}}^{-\tau_{i j}^{*}(0)} e^{\mu s}\left|e_{i}(s, x)\right|^{p} \mathrm{~d} s \\
& +e^{\mu \tau^{*}} \sum_{j=1}^{n} \tau^{*} \gamma_{i j}^{* *} \int_{-\tau^{*}}^{0} e^{\mu s}\left|e_{i}(s, x)\right|^{p} \mathrm{~d} s \\
& \left.\left.+\left|R_{i}(0, x)\right|^{p}+\frac{p}{2 \varepsilon v_{i}}\left(\epsilon_{i}(0, x)+l_{i}\right)^{2}\right] \mathrm{~d} x\right\} \\
& \leq \mathbf{E}\left\{\int _ { \Omega } \sum _ { i = 1 } ^ { n } \left[\left|e_{i}(0, x)\right|^{p}\right.\right. \\
& +\alpha_{i} e^{\mu \delta} \int_{-\delta_{i}(0)}^{0} e^{\mu s}\left|e_{i}(s, x)\right|^{p} \mathrm{~d} s \\
& +\alpha_{i}^{*} e^{\mu \delta} \int_{-\delta}^{-\delta_{i}(0)} e^{\mu s}\left|e_{i}(s, x)\right|^{p} \mathrm{~d} s \\
& +e^{\mu \tau} \max _{i=1,2, \ldots, n}\left\{\sum_{j=1}^{n} \beta_{i j}\right\} \sum_{j=1}^{n} \int_{-\tau_{i j}(0)}^{0} e^{\mu s}\left|e_{i}(s, x)\right|^{p} \mathrm{~d} s \\
& +e^{\mu \tau} \max _{i=1,2, \ldots, n}\left\{\sum_{j=1}^{n} \beta_{i j}^{*}\right\} \sum_{j=1}^{n} \int_{-\tau}^{-\tau_{i j}(0)} e^{\mu s}\left|e_{i}(s, x)\right|^{p} \mathrm{~d} s \\
& +e^{\mu \tau^{*}} \max _{i=1,2, \ldots, n}\left\{\sum_{j=1}^{n} \gamma_{i j}\right\} \sum_{j=1}^{n} \int_{-\tau_{i j}^{*}(0)}^{0} e^{\mu s}\left|e_{i}(s, x)\right|^{p} \mathrm{~d} s \\
& +e^{\mu \tau^{*}} \max _{i=1,2, \ldots, n}\left\{\sum_{j=1}^{n} \gamma_{i j}^{*}\right\} \sum_{j=1}^{n} \int_{-\tau^{*}}^{-\tau_{i j}^{*}(0)} e^{\mu s}\left|e_{i}(s, x)\right|^{p} \mathrm{~d} s \\
& +\tau^{*} e^{\mu \tau^{*}} \max _{i=1,2, \ldots, n}\left\{\sum_{j=1}^{n} \gamma_{i j}^{* *}\right\} \sum_{j=1}^{n} \int_{-\tau^{*}}^{0} e^{\mu s}\left|e_{i}(s, x)\right|^{p} \mathrm{~d} s \\
& \left.\left.+\left|R_{i}(0, x)\right|^{p}+\frac{p}{2 \varepsilon v_{i}}\left(\epsilon_{i}(0, x)+l_{i}\right)^{2}\right] d x\right\} .
\end{aligned}
$$

(40) 
Since

$$
\begin{aligned}
& \left\|\psi^{z}-\phi^{y}\right\|_{p} \\
& \quad=\left(\int_{\Omega} \sum_{i=1}^{n} \sup _{\bar{\tau} \leq \theta \leq 0}\left|\psi^{z}(\theta, x)-\phi_{i}^{y}(\theta, x)\right|^{p} \mathrm{~d} x\right)^{1 / p},
\end{aligned}
$$

then

$$
\begin{aligned}
& \int_{\Omega} \sum_{i=1}^{n} \int_{-\tau_{i j}(0)}^{0} e^{\mu s}\left|e_{i}(s, x)\right|^{p} \mathrm{~d} s \mathrm{~d} x \\
& \leq \int_{\Omega} \sum_{i=1}^{n} \int_{-\tau}^{0}\left|e_{i}(s, x)\right|^{p} \mathrm{~d} s \mathrm{~d} x \\
& \quad \leq \int_{\Omega} \sum_{i=1}^{n} \tau \sup _{-\bar{\tau} \leq s \leq 0}\left|e_{i}(s, x)\right|^{p} \mathrm{~d} x=\tau\left\|\psi^{z}-\phi^{y}\right\|_{p}^{p} .
\end{aligned}
$$

Similarly,

$$
\begin{gathered}
\int_{\Omega} \sum_{i=1}^{n}\left|e_{i}(0, x)\right|^{p} \mathrm{~d} x \leq\left\|\psi^{z}-\phi^{y}\right\|_{p}^{p}, \\
\int_{\Omega} \sum_{i=1}^{n}\left|R_{i}(0, x)\right|^{p} \mathrm{~d} x \leq\left\|\psi^{h}-\phi^{s}\right\|_{p}^{p}, \\
\int_{\Omega} \sum_{i=1}^{n} \int_{-\tau^{*}}^{0} e^{\mu s}\left|e_{i}(s, x)\right|^{p} \mathrm{~d} s \mathrm{~d} x \leq \tau^{*}\left\|\psi^{z}-\phi^{y}\right\|_{p}^{p}, \\
\int_{\Omega} \sum_{i=1}^{n} \int_{-\delta}^{-\delta_{i}(0)} e^{\mu s}\left|e_{i}(s, x)\right|^{p} \mathrm{~d} s \mathrm{~d} x \leq \delta\left\|\psi^{z}-\phi^{y}\right\|_{p}^{p}, \\
\int_{\Omega} \sum_{i=1}^{n} \int_{-\delta_{i}(0)}^{0} e^{\mu s}\left|e_{i}(s, x)\right|^{p} \mathrm{~d} s \mathrm{~d} x \leq \delta\left\|\psi^{z}-\phi^{y}\right\|_{p}^{p}, \\
\int_{\Omega} \sum_{i=1}^{n} \int_{-\delta}^{-\delta_{i}(0)} e^{\mu s}\left|e_{i}(s, x)\right|^{p} \mathrm{~d} s \mathrm{~d} x \leq \delta\left\|\psi^{z}-\phi^{y}\right\|_{p}^{p}, \\
\int_{\Omega} \sum_{i=1}^{n} \int_{-\tau^{*}}^{n} \int_{-\tau}^{-\tau_{i j}(0)} e^{\mu s}(0) \\
\int^{\mu s}\left|e_{i}(s, x)\right|^{p} \mathrm{~d} s \mathrm{~d} x \leq \tau\left\|\psi^{z}-\phi^{y}\right\|_{p}^{p},
\end{gathered}
$$

Applying (42)-(43) into (40), we have

$$
\begin{aligned}
& \mathbf{E}\{V(0)\} \leq\left[1+\alpha_{i} \delta e^{\mu \delta}+\alpha_{i}^{*} \delta e^{\mu \delta}\right. \\
& +n \tau e^{\mu \tau} \max _{i=1,2, \ldots, n}\left\{\sum_{j=1}^{n} \beta_{i j}\right\}+n \tau e^{\mu \tau} \max _{i=1,2, \ldots, n}\left\{\sum_{j=1}^{n} \beta_{i j}^{*}\right\} \\
& +n \tau^{*} e^{\mu \tau^{*}} \max _{i=1,2, \ldots, n}\left\{\sum_{j=1}^{n} \gamma_{i j}\right\} \\
& +n \tau^{*} e^{\mu \tau^{*}} \max _{i=1,2, \ldots, n}\left\{\sum_{j=1}^{n} \gamma_{i j}^{*}\right\} \\
& \left.+n \tau^{*^{2}} e^{\mu \tau^{*}} \max _{i=1,2, \ldots, n}\left\{\sum_{j=1}^{n} \gamma_{i j}^{* *}\right\}+\frac{N}{\left\|\psi^{z}-\phi^{y}\right\|_{p}^{p}}\right] \\
& \cdot \mathbf{E}\left\{\left\|\psi^{z}-\phi^{y}\right\|_{p}^{p}\right\}+\mathbf{E}\left\{\left\|\psi^{h}-\phi^{s}\right\|_{p}^{p}\right\} \\
& =M \mathbf{E}\left\{\left\|\psi^{z}-\phi^{y}\right\|_{p}^{p}\right\}+M^{*} \mathbf{E}\left\{\left\|\psi^{h}-\phi^{s}\right\|_{p}^{p}\right\},
\end{aligned}
$$

where

$$
\begin{aligned}
M^{*}= & 1, \\
N= & \int_{\Omega} \sum_{i=1}^{n} \frac{p}{2 \varepsilon \nu_{i}}\left(\epsilon_{i}(0, x)+l_{i}\right)^{2} \mathrm{~d} x \\
M= & +\alpha_{i} \delta e^{\mu \delta}+\alpha_{i}^{*} \delta e^{\mu \delta}+n \tau e^{\mu \tau} \max _{i=1,2, \ldots, n}\left\{\sum_{j=1}^{n} \beta_{i j}\right\} \\
& +n \tau e^{\mu \tau} \max _{i=1,2, \ldots, n}\left\{\sum_{j=1}^{n} \beta_{i j}^{*}\right\} \\
& +n \tau^{*} e^{\mu \tau^{*}} \max _{i=1,2, \ldots, n}\left\{\sum_{j=1}^{n} \gamma_{i j}\right\} \\
& +n \tau^{*} e^{\mu \tau^{*}} \max _{i=1,2, \ldots, n}\left\{\sum_{j=1}^{n} \gamma_{i j}^{*}\right\} \\
& +n \tau^{*^{2}} e^{\mu \tau^{*}} \max _{i=1,2, \ldots, n}\left\{\sum_{j=1}^{n} \gamma_{i j}^{* *}\right\}+\frac{N}{\left\|\psi^{z}-\phi^{y}\right\|_{p}^{p}} \\
& 1 .
\end{aligned}
$$

Therefore,

$\mathbf{E}\{V(t)\} \leq \mathbf{E}\{V(0)\}$

$$
\leq M \mathbf{E}\left\{\left\|\psi^{z}-\phi^{y}\right\|_{p}^{p}\right\}+M^{*} \mathbf{E}\left\{\left\|\psi^{h}-\phi^{s}\right\|_{p}^{p}\right\} .
$$


Further, we obtain

$$
\begin{aligned}
& \mathbf{E}\{V(t)\} \geq \mathbf{E}\left\{\int_{\Omega} \sum_{i=1}^{n}\left[V_{i}(t, x)+e^{\mu t}\left|R_{i}(t, x)\right|^{p}\right] \mathrm{d} x\right\} \\
& =e^{\mu t} \mathbf{E}\left\{\int_{\Omega} \sum_{i=1}^{n}\left[\left|z_{i}(t, x)-y_{i}(t, x)\right|^{p}+\left|h_{i}(t, x)-s_{i}(t, x)\right|^{p}\right] \mathrm{d} x\right\} \\
& =e^{\mu t} \mathbf{E}\left\{\|z(t, x)-y(t, x)\|^{p}\right\}+e^{\mu t} \mathbf{E}\left\{\|h(t, x)-s(t, x)\|^{p}\right\} .
\end{aligned}
$$

From (46) and (47), we have

$$
\begin{aligned}
\mathbf{E}\left\{\|z(t, x)-y(t, x)\|^{p}\right\}+\mathbf{E}\left\{\|h(t, x)-s(t, x)\|^{p}\right\} \\
\leq M \mathbf{E}\left\{\left\|\psi^{z}-\phi^{y}\right\|_{p}^{p}\right\} e^{-\mu t} \\
+M^{*} \mathbf{E}\left\{\left\|\psi^{h}-\phi^{s}\right\|_{p}^{p}\right\} e^{-\mu t} .
\end{aligned}
$$

Hence, the nonlinear couple neural networks (9) and (5) can be exponentially synchronized under the adaptive feedback controller (11) and (12) based on p-norm. The proof of Theorem 8 is complete.

Remark 9. It is the first time to consider the combined effects of time-varying leakage delays, the discrete time-varying delay, the distributed time-varying delay, stochastic perturbation, and spatial diffusion on the exponential synchronization of competitive neural networks under an adaptive feedback controller. The neural networks discussed in $[6,7,31]$ are the special cases of the model in this paper. From this point, our results are more general.

Remark 10. In Theorem 8, the sufficient conditions are derived to achieve the adaptive synchronization for the proposed competitive neural networks. Compared with the adaptive synchronization criteria given in [7], the conditions obtained in Theorem 8 depend on not only the timescale $\varepsilon$ but also the controller parameter $\mu$. It is beneficial to design an adaptive controller to realize the adaptive synchronization for the neural networks. Therefore, the criteria derived in this paper have wider application.

\section{Numerical Simulations}

In this section, some numerical simulation examples demonstrate the main results in Theorem 8 . form

In system (5), we choose $n=2$. Then system (5) takes the

$$
\begin{aligned}
\text { STM: } \varepsilon \frac{\partial y_{i}(t, x)}{\partial t}= & D_{i} \Delta y_{i}(t, x)-c_{i} y_{i}(t-\delta(t), x) \\
& +\sum_{j=1}^{n} a_{i j} f_{j}\left(y_{j}(t, x)\right) \\
& +\sum_{j=1}^{n} b_{i j} f_{j}\left(y_{j}(t-\tau(t), x)\right)
\end{aligned}
$$

$$
\begin{aligned}
& +\sum_{j=1}^{n} d_{i j} \int_{t-\tau^{*}(t)}^{t} f_{j}\left(y_{j}(s, x)\right) \mathrm{d} s \\
& +H_{\mathrm{i}} s_{i}(t, x), \\
\operatorname{LTM}: \frac{\partial s_{i}(t, x)}{\partial t}= & -K_{i} s_{i}(t, x)+f_{i}\left(y_{i}(t, x)\right),
\end{aligned}
$$

where $f_{j}\left(u_{j}(t, x)\right)=\tanh \left(u_{j}(t, x)\right), \delta(t)=0.4+0.3 \cos t$, $\tau(t)=0.5+0.4 \cos t$, and $\tau^{*}(t)=0.3+0.2 \sin t$. The parameters of (49) are assumed as follows: $\varepsilon=0.5, D_{1}=0.025, D_{2}=$ $0.025, c_{1}=0.35, c_{2}=0.25, a_{11}=0.625, a_{12}=-0.3, a_{21}=$ $-0.225, a_{22}=-0.3, b_{11}=-0.475, b_{12}=-0.425, b_{21}=0.375$, $b_{22}=0.475, d_{11}=-0.375, d_{12}=-0.325, d_{21}=0.6, d_{22}=0.8$, $H_{1}=0.375, H_{2}=0.375, K_{1}=1, K_{2}=1$, and $x \in \Omega=[-5,5]$. The initial conditions of system (49) are chosen as

$$
\begin{aligned}
& y_{1}(s, x)=0.1 \cos \left(\frac{x+5}{10} \pi\right), \\
& y_{2}(s, x)=0.2 \cos \left(\frac{x+5}{10} \pi\right), \\
& s_{1}(s, x)=0.3 \cos \left(\frac{x+5}{10} \pi\right), \\
& s_{2}(s, x)=0.4 \cos \left(\frac{x+5}{10} \pi\right),
\end{aligned}
$$

where $(s, x) \in[-0.9,0] \times \Omega$.

Numerical simulation illustrates that the reactiondiffusion neural network (49) with boundary condition (6) and the initial condition (50) exhibits a chaotic behavior (see Figure 1).

The noise-perturbed response system is described by

STM: $\mathrm{d} z_{i}(t, x)=\frac{1}{\varepsilon}\left[D_{i} \Delta z_{i}(t, x)-c_{i} z_{i}(t-\delta(t), x)\right.$

$$
\begin{aligned}
& +\sum_{j=1}^{n} a_{i j} f_{j}\left(z_{j}(t, x)\right)+\sum_{j=1}^{n} b_{i j} f_{j}\left(z_{j}(t-\tau(t), x)\right) \\
& +\sum_{j=1}^{n} d_{i j} \int_{t-\tau^{*}(t)}^{t} f_{j}\left(z_{j}(s, x)\right) \mathrm{d} s+H_{i} h_{i}(t, x)+u_{i}(t,
\end{aligned}
$$

$$
\begin{array}{r}
x)] \mathrm{d} t+\sum_{j=1}^{n} \sigma_{i j}\left(e_{j}(t, x), e_{j}\left(t-\delta_{i}(t), x\right),\right. \\
\left.e_{j}\left(t-\tau_{i j}(t), x\right), e_{j}\left(t-\tau_{i j}^{*}(t), x\right)\right) \mathrm{d} \omega_{j}(t), \\
\mathrm{LTM}: \frac{\partial h_{i}(t, x)}{\partial t}=-K_{i} h_{i}(t, x)+f_{i}\left(z_{i}(t, x)\right),
\end{array}
$$



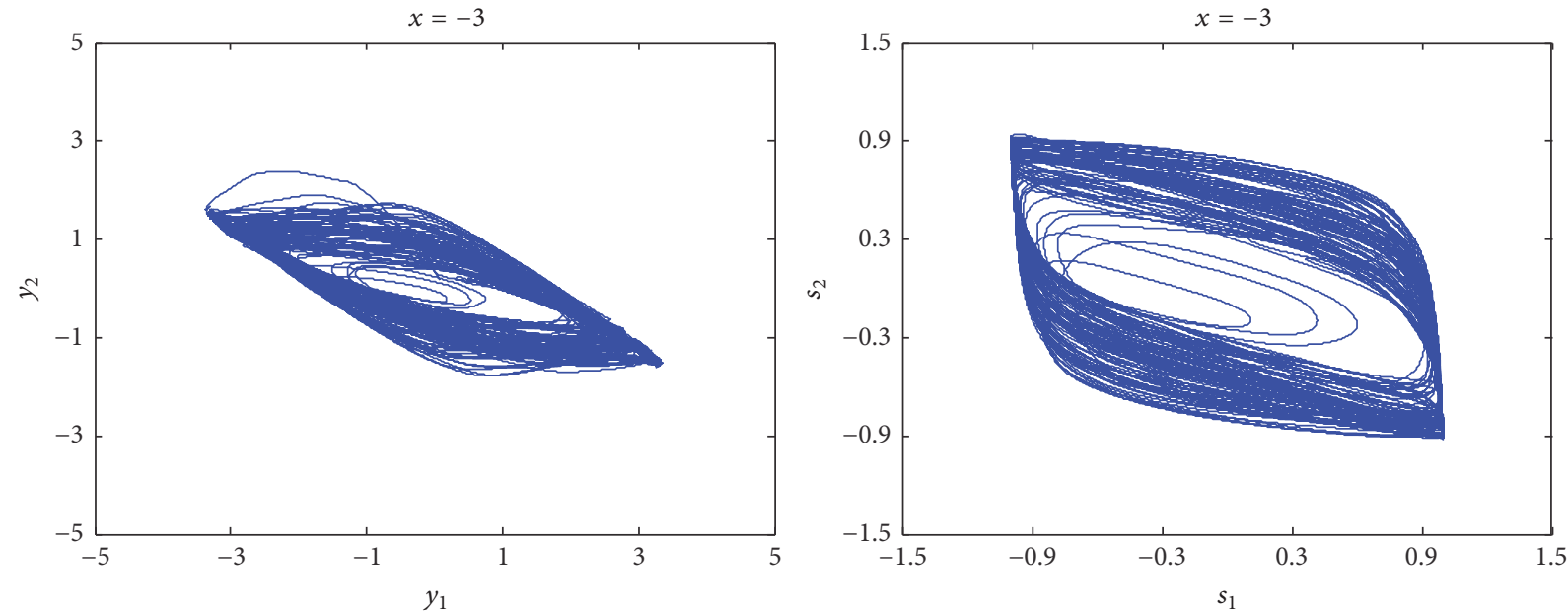

FIGURE 1: Chaotic behaviors of competitive neural networks (49).
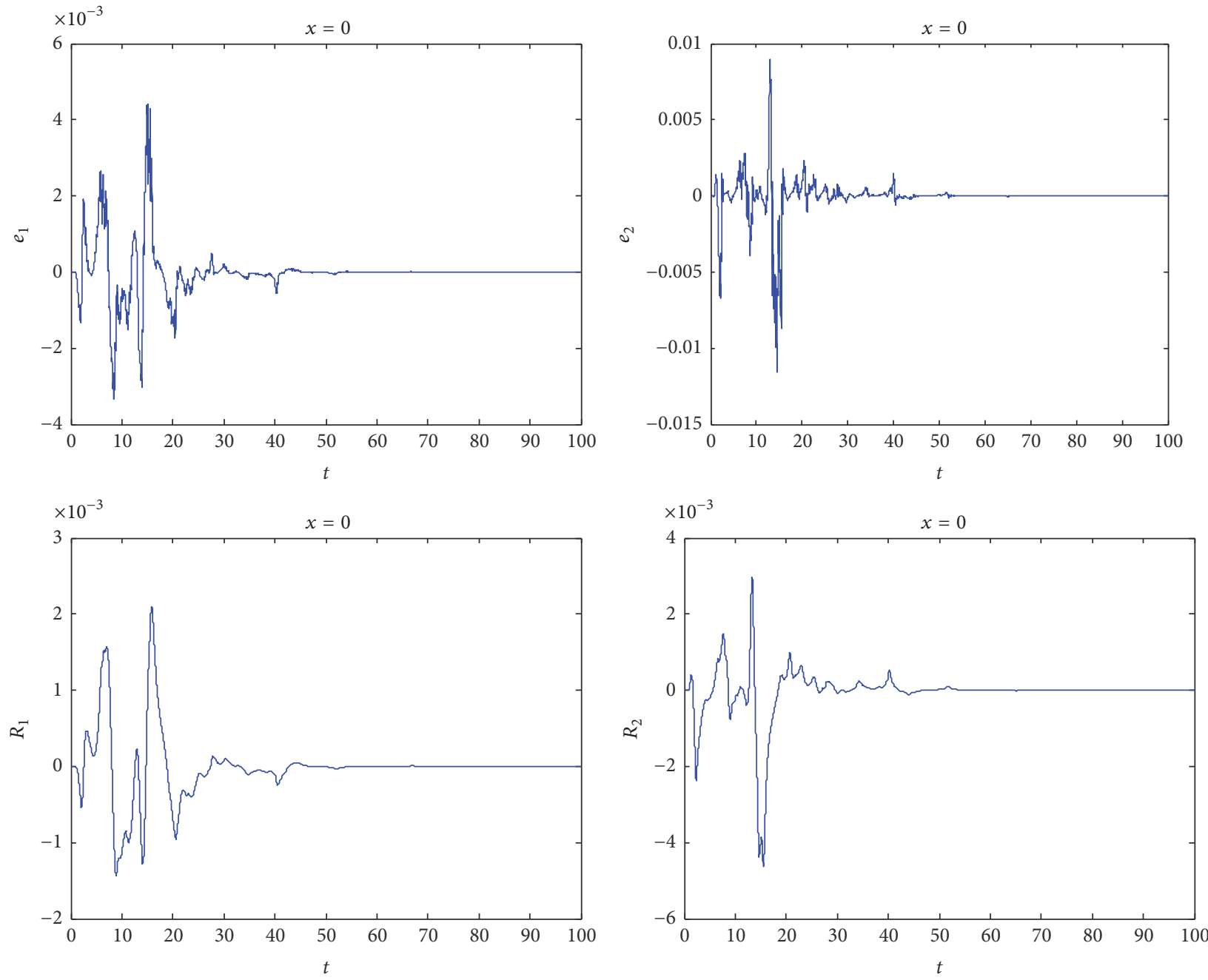

FIGURE 2: Asymptotical behaviors of the synchronization errors between systems (49) and (51). 

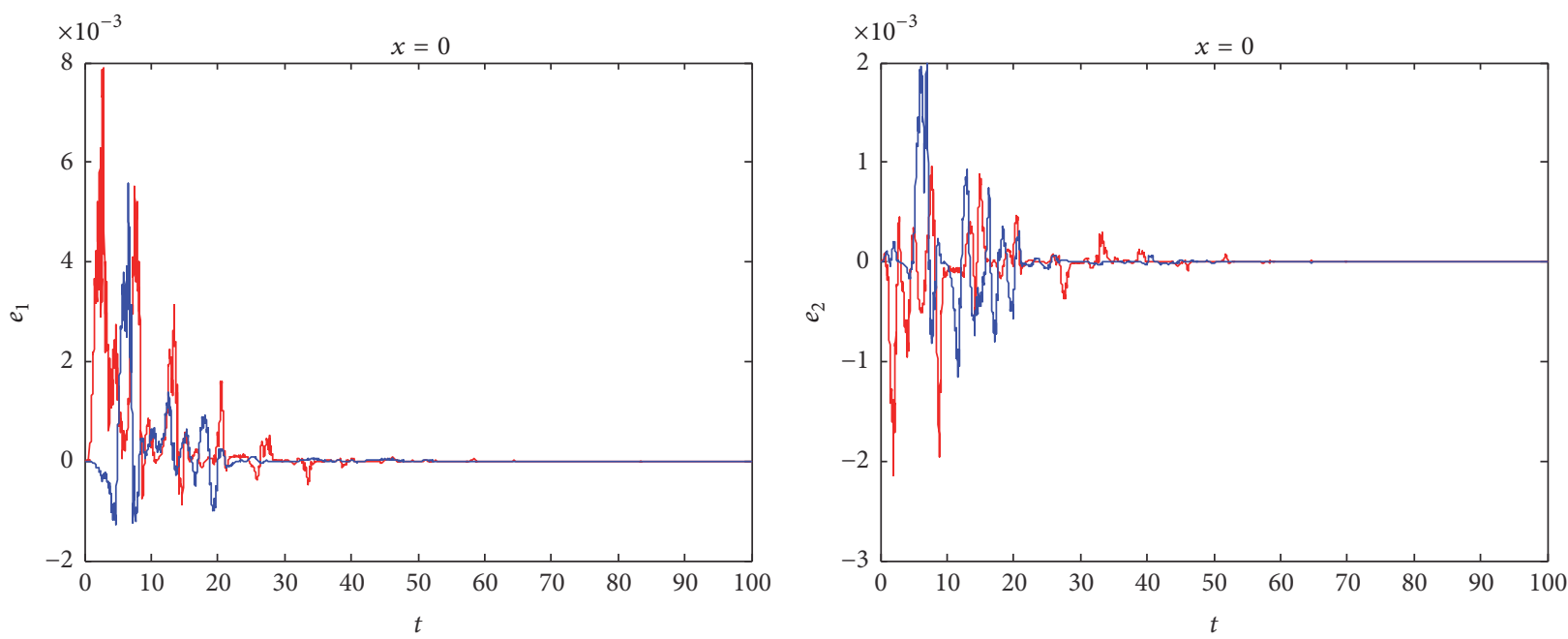

$-\varepsilon=0.27$

$-\varepsilon=0.27$

$\varepsilon=1$
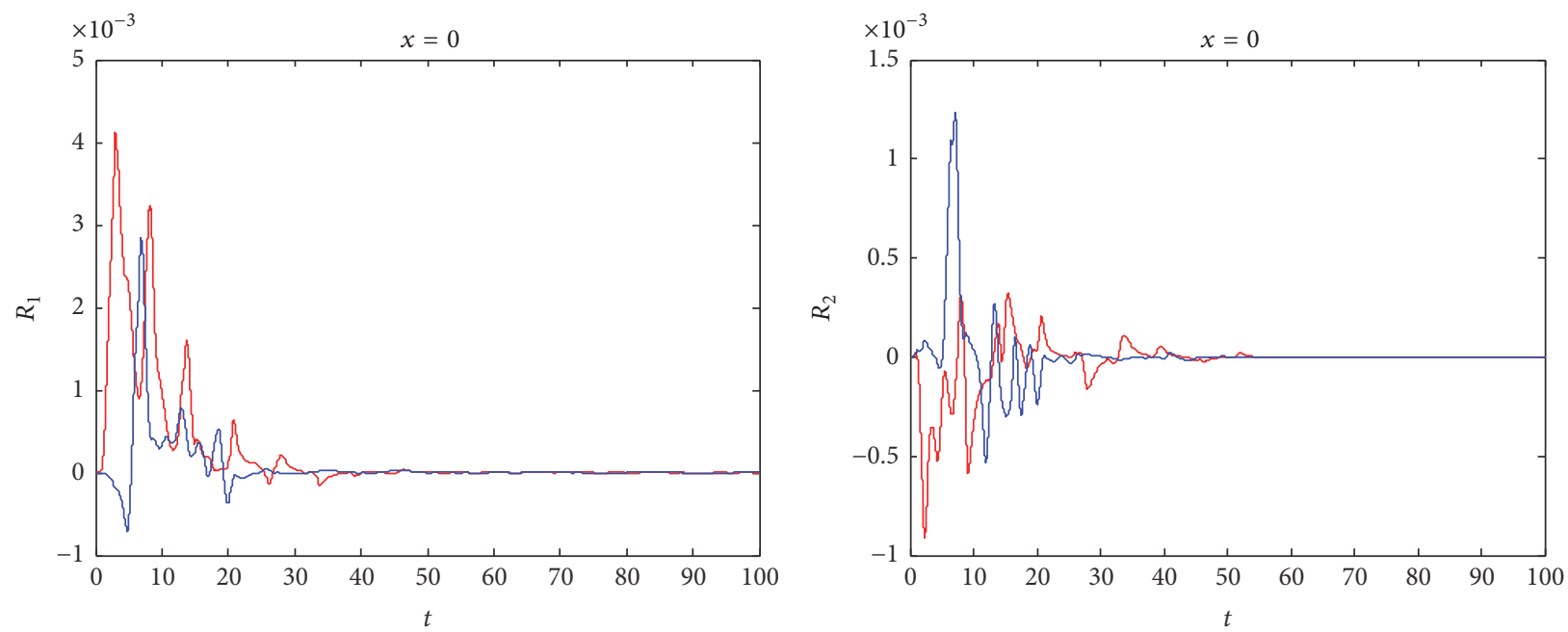

$-\varepsilon=0.27$

$\varepsilon=0.27$

$\varepsilon \varepsilon=1$

$-\varepsilon=1$

FIgURE 3: Asymptotical behaviors of the synchronization errors with differential timescale $\varepsilon$.

where

$$
\begin{aligned}
\sigma_{11}= & 0.1 e_{1}(t, x)+0.2 e_{1}(t-\tau(t), x) \\
& +0.1 e_{1}(t-\delta(t), x)+0.3 e_{1}\left(t-\tau^{*}(t), x\right), \\
\sigma_{12}= & 0, \\
\sigma_{21}= & 0, \\
\sigma_{22}= & 0.2 e_{2}(t, x)+0.1 e_{2}(t-\tau(t), x) \\
& +0.3 e_{2}(t-\delta(t), x)+0.1 e_{2}\left(t-\tau^{*}(t), x\right) .
\end{aligned}
$$

The adaptive controller is

$$
u_{i}(t, x)=\epsilon_{i} e_{i}(t, x) \text {, }
$$

$$
\frac{\partial \epsilon_{i}}{\partial t}=-\left|e_{i}(t, x)\right|^{2} e_{i}^{0.1 t}
$$

The initial conditions for the response system (51) are chosen as

$$
\begin{aligned}
& z_{1}(s, x)=0.1 \cos \left(\frac{x+5}{10} \pi\right), \\
& z_{2}(s, x)=0.2 \cos \left(\frac{x+5}{10} \pi\right), \\
& h_{1}(s, x)=0.3 \cos \left(\frac{x+5}{10} \pi\right), \\
& h_{2}(s, x)=0.4 \cos \left(\frac{x+5}{10} \pi\right),
\end{aligned}
$$

where $(s, x) \in[-0.9,0] \times \Omega$. 

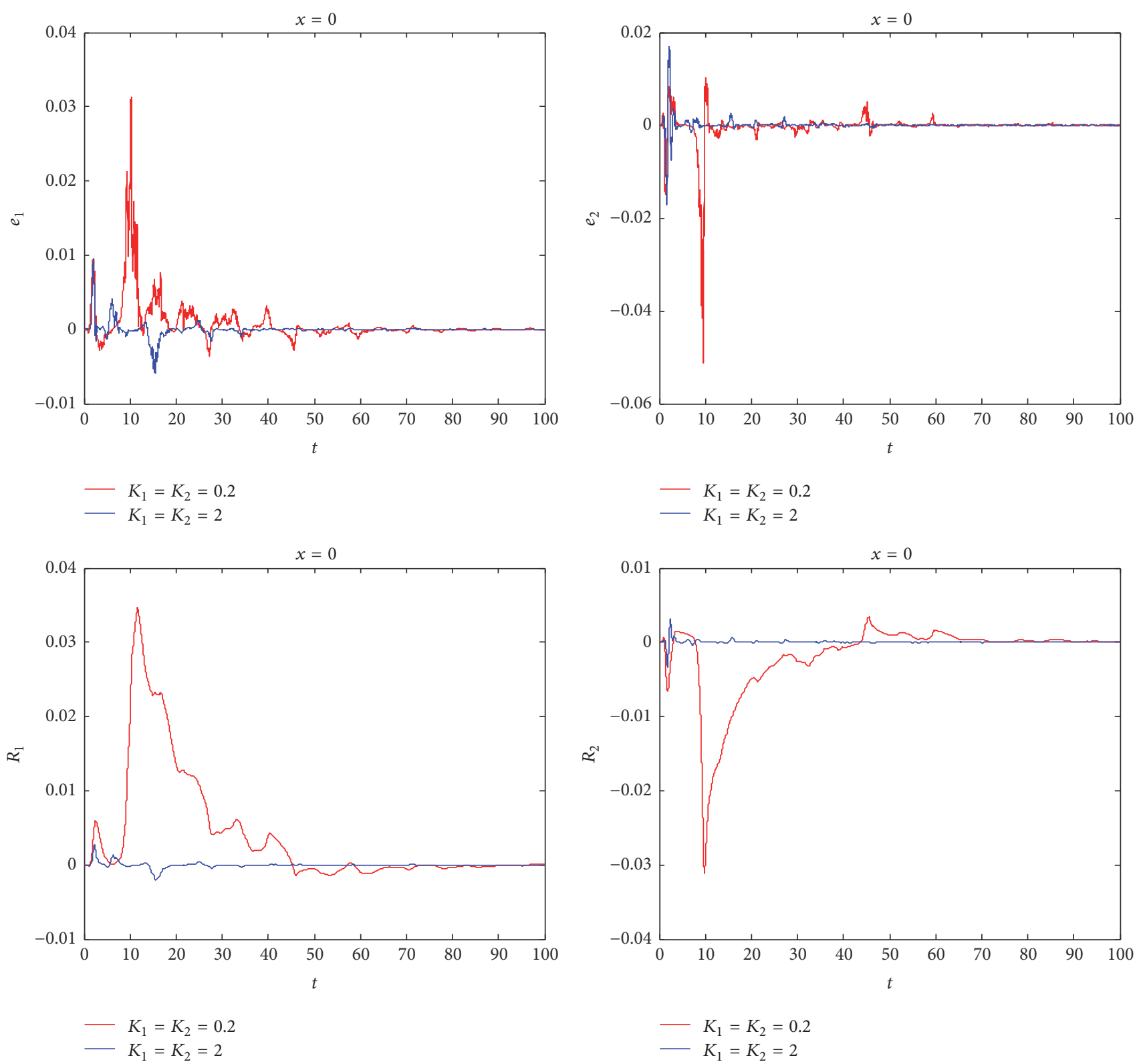

FIGURE 4: Asymptotical behaviors of the synchronization errors with differential disposable scaling constants $K_{i}$.

Evidently, $L_{1}=L_{2}=1, \rho^{\prime}=0.3<1, \varrho^{\prime}=0.4<1$, and $\varrho^{* \prime}=0.2<1$. Let $p=2, \varepsilon=0.5, \xi_{1}=\xi_{2}=0.1$, and $\xi_{1}^{*}=\xi_{2}^{*}=1$. By simple computation, it is easy to verify that assumptions $\left(\mathrm{H}_{1}\right)-\left(\mathrm{H}_{6}\right)$ are satisfied. According to Theorem 8, the drive system (49) and the response system (51) are exponentially synchronized based on $p$-norm. Numerical simulation illustrates our results (see Figure 2).

Remark 11. The conclusions given in Theorem 8 show that the adaptive synchronization criteria for competitive neural networks are dependent on the timescale $\varepsilon$, the disposable scaling constant $K_{i}$, and the external stimulus $H_{i}$. When $\varepsilon$ increases, and $K_{i}$ increases or $H_{i}$ decreases, respectively, assumption $\left(\mathrm{H}_{6}\right)$ can be satisfied more easily, and the adaptive synchronization of the competitive neural networks is more easily realized. Dynamical behaviors of the synchronization errors between systems (49) and (51) with the differential timescale, disposable scaling constant, and external stimulus, respectively, are shown in Figures 3-5.

Remark 12. By (48), it is clear to see that the controller parameter $\mu$ denotes the rate of the synchronization. That is, the larger the controller parameter $\mu$ is, the faster systems (49) and (51) realize synchronization. Hence, our results are consistent with the practical situation. Dynamical behaviors of the synchronization errors between systems (49) and (51) with differential controller parameter $\mu$ are shown in Figure 6. 

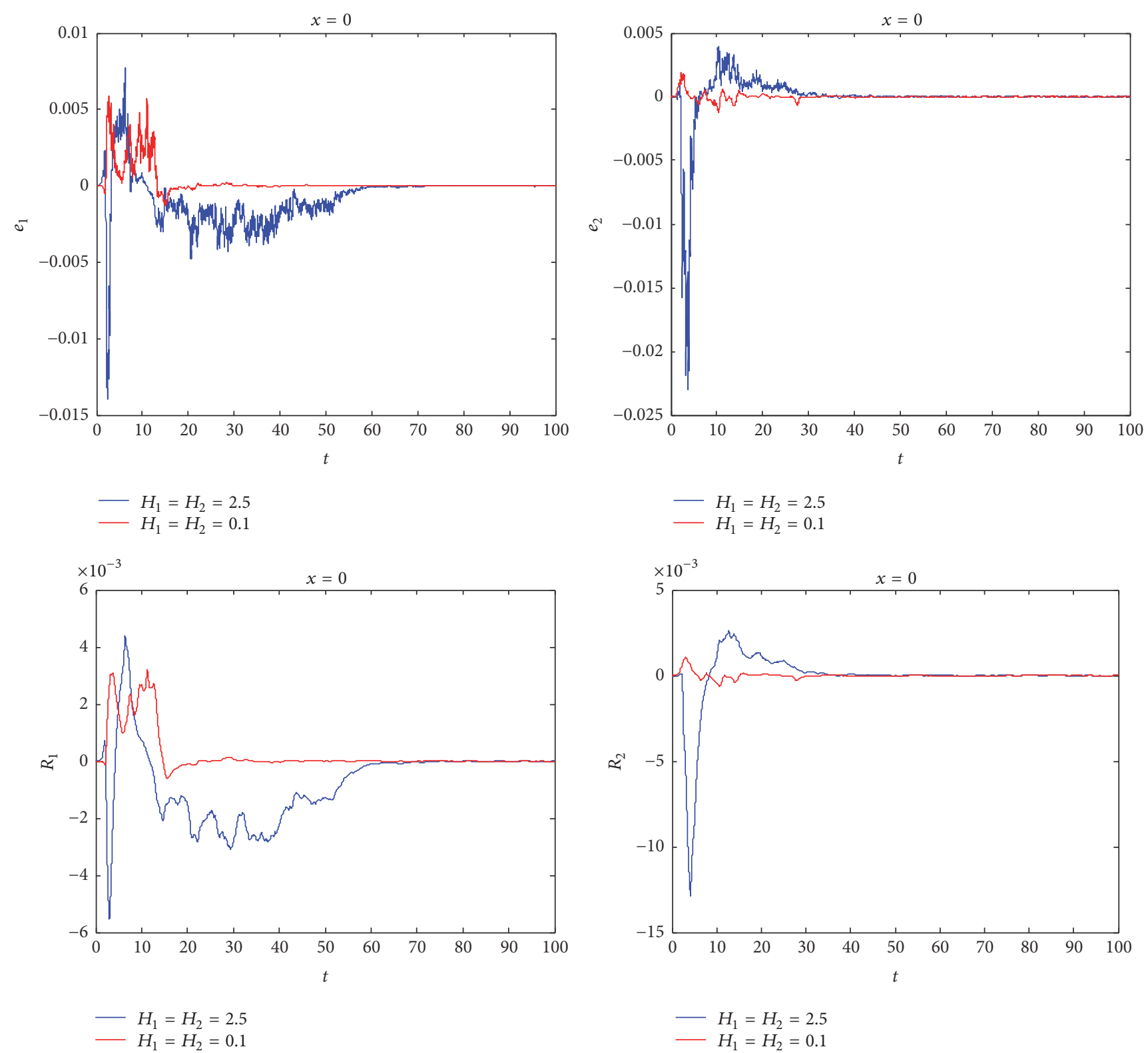

Figure 5: Asymptotical behaviors of the synchronization errors with differential external stimulus constants $H_{i}$.

The parameter $v_{i}$ is another controller parameter in the feedback controller (11). By numerical simulations, we can see that it is beneficial for competitive neural networks to realize the synchronization by increasing controller parameter $v_{i}$. Dynamical behaviors of the synchronization errors between systems (49) and (51) with differential controller parameter $v_{i}$ are shown in Figure 7. However, we cannot prove it. It is an interesting open problem to research.

Remark 13. In many cases, two-neuron networks show the same behavior as large-size networks and many research methods used in two-neuron networks can be applied to large-size networks. Therefore, a two-neuron network can be used as an example to improve our understanding of our theoretical results. In addition, the parameter values are selected randomly to ensure that neural networks (49) exhibit a chaotic behavior.

\section{Conclusion}

In this paper, an adaptive feedback controller was designed to achieve the exponential synchronization for stochastic competitive neural networks with spatial diffusion, time-varying leakage delays, and discrete and distributed time-varying delays based on $p$-norm. Evidently, the model discussed in this paper is more general than those correspondent models when the delays are constant delays. By constructing the Lyapunov functional and using and the stochastic analysis 

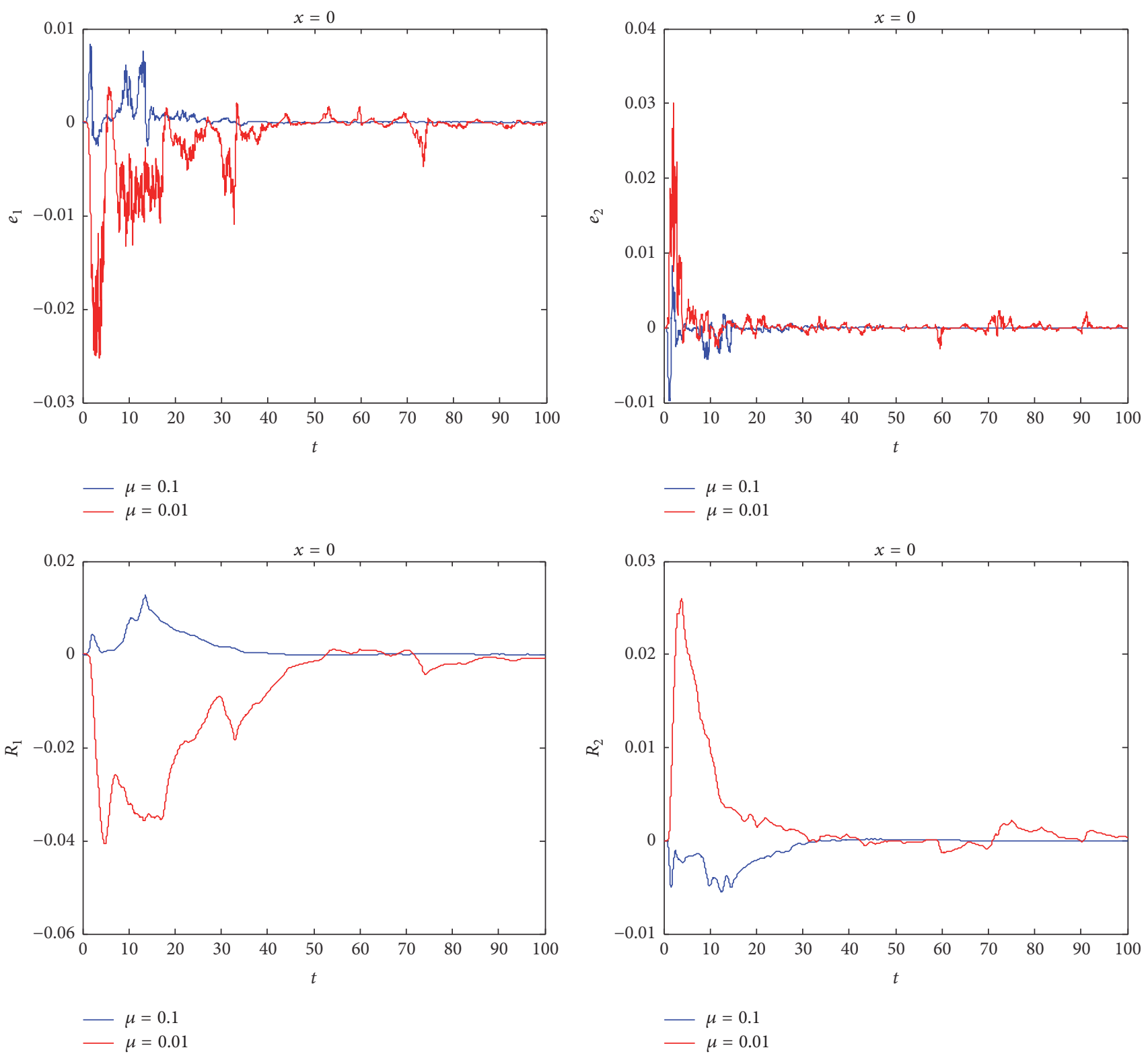

FIgURE 6: Asymptotical behaviors of the synchronization errors with differential controller parameter $\mu$.

theory, the novel exponential synchronization criteria dependent on the timescale $\varepsilon$, external stimulus constants $H_{i}$, disposable scaling constants $K_{i}$, and controller parameter $\mu$ were obtained. By theory analysis, it was shown that competitive neural networks can achieve exponential synchronization more easily by increasing the timescale and disposable scaling constants or reducing disposable scaling constants, respectively. Numerical examples and their simulations are given to show the effectiveness of the obtained results.

Figures 8 and 9 show that it is beneficial for competitive neural networks with reaction-diffusion terms to realize the synchronization by increasing diffusion coefficients $D_{i}$ or decreasing diffusion space $x$, respectively. However, the exponential synchronization criteria obtained in this paper are independent of the diffusion coefficients and the diffusion space. They cannot reflect the influence of the diffusion coefficients and diffusion space on synchronization, which limits the application scopes of the results. Therefore, we will investigate that in our future work.

\section{Appendix}

Proof of Lemma 6. According to the eigenvalue theory of elliptic operators, the Laplacian $-\Delta$ on $\Omega$ with the Neumann boundary conditions is a self-adjoint operator with compact inverse, so there exists a sequence of nonnegative eigenvalues $0=\lambda_{0}<\lambda_{1}<\lambda_{2}<\cdots,\left(\lim _{i \rightarrow \infty} \lambda_{i}=+\infty\right)$ as well as a sequence of corresponding eigenfunctions $\vartheta_{0}(x)$, 

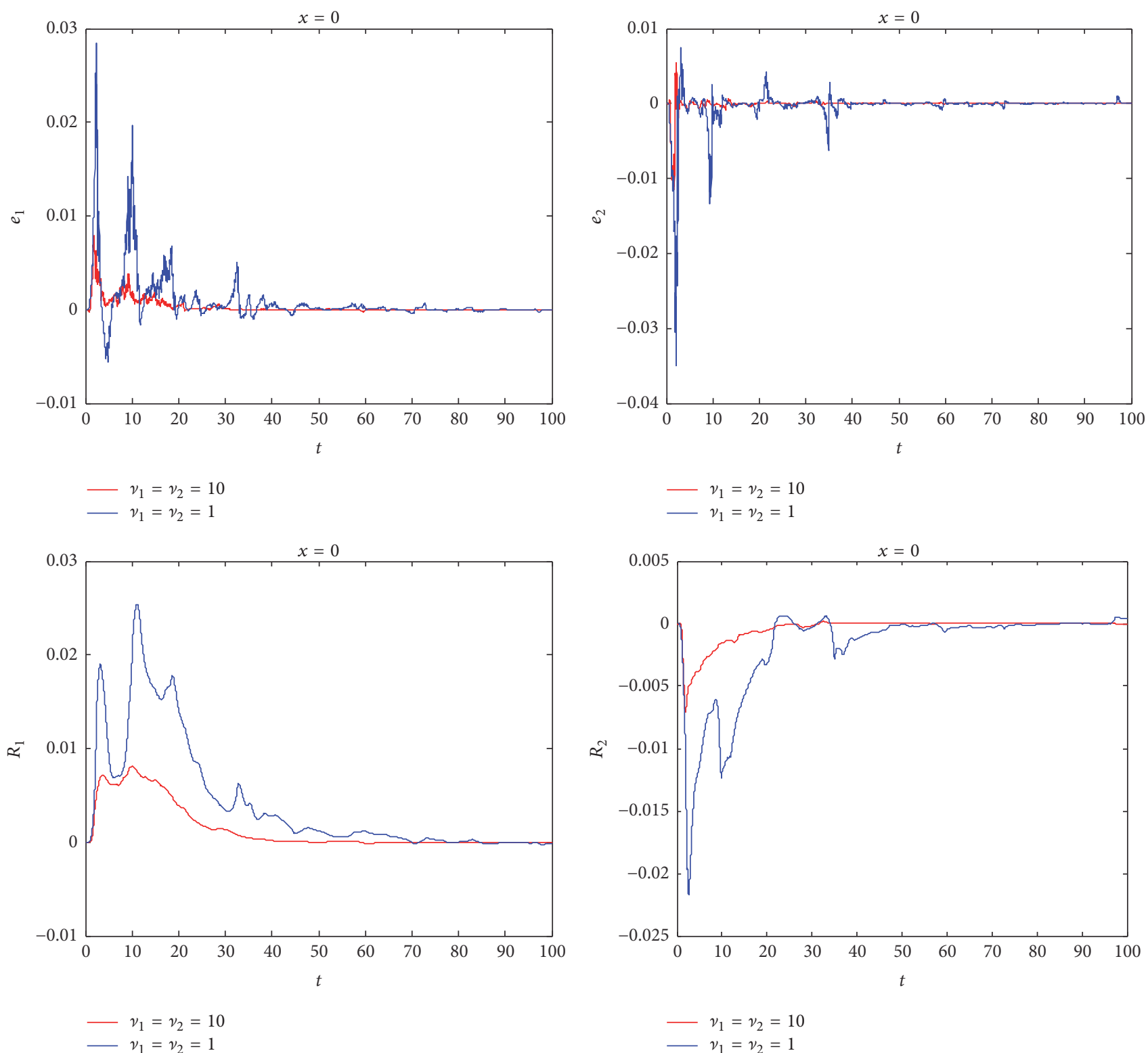

FIGURE 7: Asymptotical behaviors of the synchronization errors with differential controller parameter $v_{i}$.

$\vartheta_{1}(x), \vartheta_{2}(x), \ldots$ for the Neumann boundary problem (29); that is,

$$
\begin{aligned}
\lambda_{0} & =0, \quad \vartheta_{0}(x)=1, \\
-\Delta \vartheta_{m}(x) & =\lambda_{m} \vartheta_{m}(x), \quad \text { in } \Omega, \\
\frac{\partial \vartheta_{m}(x)}{\partial \mathbf{n}} & =0, \quad \text { on } \partial \Omega .
\end{aligned}
$$

Multiply the second equation of (A.1) by $\vartheta_{m}^{p-1}(x)(m=$ $1,2, \ldots$ ) and integrate $\Omega$. By Green formula (27), we obtain

$$
\begin{gathered}
\lambda_{m} \int_{\Omega} \vartheta_{m}^{p}(x) \mathrm{d} x=-\int_{\Omega} \vartheta_{m}^{p-1}(x) \Delta \vartheta_{m}(x) \mathrm{d} x \\
\quad=-\int_{\partial \Omega} \vartheta_{m}^{p-1}(x) \frac{\partial \vartheta_{m}(x)}{\partial \mathbf{n}} \mathrm{d} s
\end{gathered}
$$

$$
\begin{aligned}
& +\int_{\Omega}\left(\nabla \vartheta_{m}^{p-1}(x)\right)^{T} \nabla \vartheta_{m}(x) \mathrm{d} x=\int_{\Omega}(p-1) \\
& \cdot \vartheta_{m}^{p-2}(x)\left[\left(\frac{\partial \vartheta_{m}(x)}{\partial x_{1}}\right)^{2}+\left(\frac{\partial \vartheta_{m}(x)}{\partial x_{2}}\right)^{2}+\cdots\right. \\
& \left.+\left(\frac{\partial \vartheta_{m}(x)}{\partial x_{l^{*}}}\right)^{2}\right] \mathrm{d} x=(p-1) \int_{\Omega} \vartheta_{m}^{p-2}(x)
\end{aligned}
$$$$
\cdot\left|\nabla \vartheta_{m}(x)\right|^{2} \mathrm{~d} x
$$

It is easy to show that (A.2) is also true for $m=0$. 

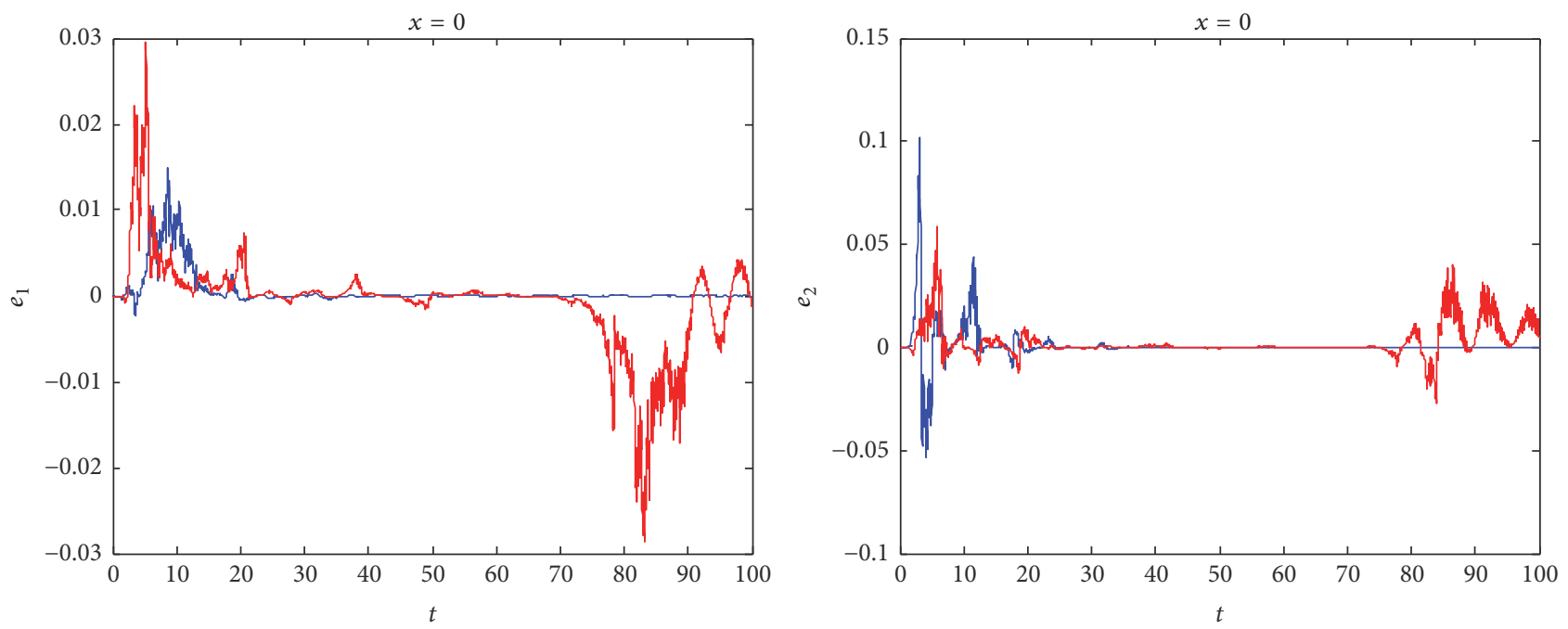

$-D_{1}=D_{2}=0.2$

$D_{1}=D_{2}=0.2$

$-D_{1}=D_{2}=0.01$

$-D_{1}=D_{2}=0.01$
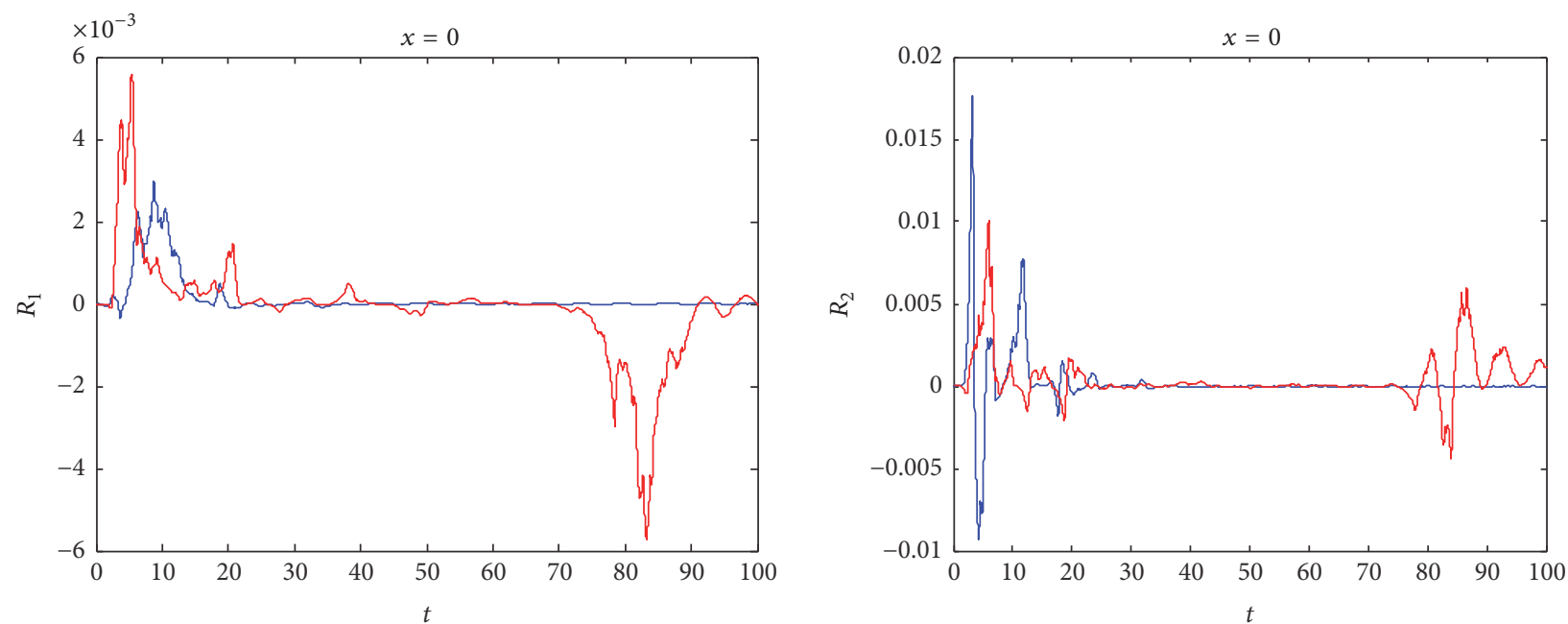

$-D_{1}=D_{2}=0.2$

$D_{1}=D_{2}=0.01$

$-D_{1}=D_{2}=0.2$

- $D_{1}=D_{2}=0.01$

FIGURE 8: Asymptotical behaviors of the synchronization errors with differential diffusion coefficients $D_{i}$.

The sequence of eigenfunctions $\left\{\vartheta_{i}(x)\right\}_{i \geq 0}$ generates an orthonormal basis of $L^{2}(\Omega)$. Hence, for any $\varphi(x) \in L^{2}(\Omega)$, there exists a sequence of constant $\left\{c_{m}\right\}_{m \geq 0}$ such that

$$
\varphi(x)=\sum_{m=0}^{\infty} c_{m} \vartheta_{m}(x) .
$$

It follows from (A.2) and (A.3) that

$$
\begin{aligned}
& \int_{\Omega}|\varphi(x)|^{p} \mathrm{~d} x \leq \int_{\Omega} \sum_{m=0}^{\infty}\left|c_{m} \vartheta_{m}(x)\right|^{p} \mathrm{~d} x \\
& \leq \frac{p-1}{\lambda_{1}} \int_{\Omega} \sum_{m=0}^{\infty}\left|c_{m} \vartheta_{m}(x)\right|^{p-2}\left|c_{m} \nabla \vartheta_{m}(x)\right|^{2} \mathrm{~d} x
\end{aligned}
$$

$$
\begin{aligned}
& \leq \frac{p-1}{\lambda_{1}} \int_{\Omega} \sum_{m=0}^{\infty}\left|c_{m} \vartheta_{m}(x)\right|^{p-2} \sum_{m=0}^{\infty}\left|c_{m} \nabla \vartheta_{m}(x)\right|^{2} \mathrm{~d} x \\
& =\frac{p-1}{\lambda_{1}} \int_{\Omega}|\varphi(x)|^{p-2}|\nabla \varphi(x)|^{2} \mathrm{~d} x .
\end{aligned}
$$

The proof of Lemma 6 is complete.

\section{Conflicts of Interest}

The authors declare that there are no conflicts of interest regarding the publication of this paper. 

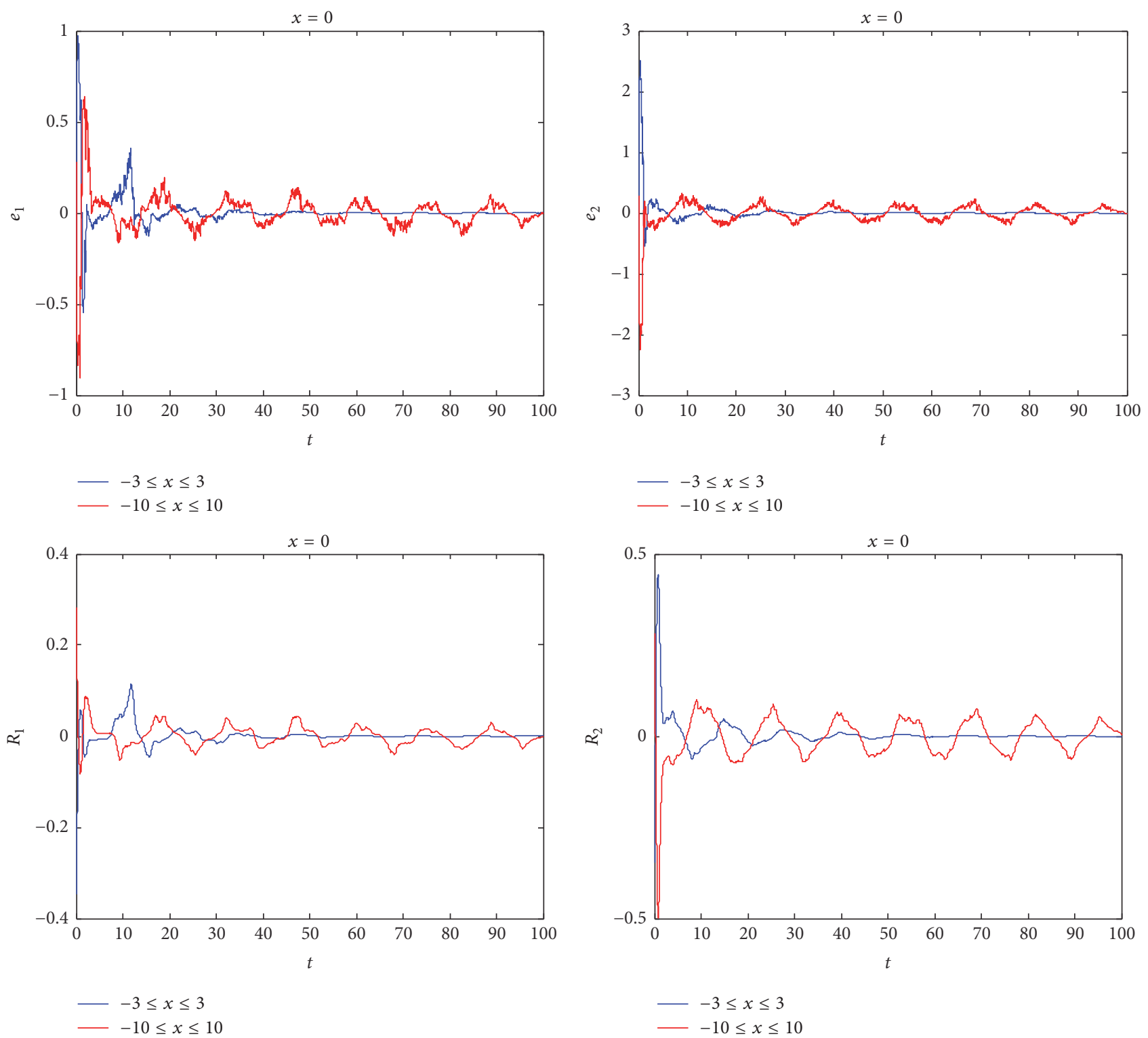

FIGURE 9: Asymptotical behaviors of the synchronization errors with differential diffusion space.

\section{Acknowledgments}

This work was supported by the Science and Technology Support Program of Hebei Academy of Sciences (17606), the Science and Technology Support Program of Hebei Province (16290106D), and the National Natural Science Foundation of China (61305076).

\section{References}

[1] A. Meyer-Base, F. Ohl, and H. Scheich, "Singular perturbation analysis of competitive neural networks with different time scales," Neural Computation, vol. 8, no. 8, pp. 1731-1742, 1996.

[2] X. Yang, J. Cao, Y. Long, and W. Rui, "Adaptive lag synchronization for competive neural networks with mixed delays and uncertain hybrid perturbations," IEEE Transactions on Neural Networks, vol. 21, no. 10, pp. 1656-1667, 2010.
[3] X. Yang, C. Huang, and J. Cao, "An LMI approach for exponential synchronization of switched stochastic competitive neural networks with mixed delayed," Neural Computing and Applications, vol. 21, no. 8, pp. 2033-2047, 2012.

[4] Q. Gan, R. Xu, and X. Kang, "Synchronization of unknown chaotic delayed competitive neural networks with different time-scales based on adaptive control and parameter identification," Nonlinear Dynamics, vol. 67, no. 3, pp. 1893-1902, 2012.

[5] Y. Li, X. Yang, and L. Shi, "Finite-time synchronization for competitive neural networks with mixed delays and non-identical perturbations," Neurocomputing, vol. 185, pp. 242-253, 2016.

[6] Y. Shi and P. Zhu, "Synchronization of stochastic competitive neural networks with different timescales and reactiondiffusion terms," Neural Computation, vol. 26, no. 9, pp. 20052024, 2014. 
[7] Q. Gan, R. Hu, and Y. Liang, "Adapative synchronization for stochastic competitive neural networks with mixed timevarying delays," Communications in Nonlinear Science and Numerical Simulation, vol. 17, no. 9, pp. 3708-3718, 2012.

[8] B. Kosko, Neural Networks and Fuzzy Systems: A Dynamical Systems Approach to Machine Intelligence, Prentice Hall, New Delhi, 1992.

[9] S. Haykin, Neural Networks, Prentice Hall, New Jersey, 1999.

[10] K. Gopalsamy, "Leakage delays in BAM," Journal of Mathematical Analysis and Applications, vol. 325, no. 2, pp. 1117-1132, 2007.

[11] C. Zheng, Y. Wang, and Z. Wang, "Stability analysis of stochastic fuzzy Markovian jumping neural networks with leakage delay under impulsive perturbations," Journal of the Franklin Institute, vol. 351, no. 3, pp. 1728-1755, 2014.

[12] R. Raja, Q. Zhu, S. Senthilraj, and R. Samidurai, "Improved stability analysis of uncertain neutral type neural networks with leakage delays and impulsive effects," Applied Mathematics and Computation, vol. 266, Article ID 21301, pp. 1050-1069, 2015.

[13] M. Kalpana and P. Balasubramaniam, "Asymptotical state eatimation of fuzzy cellular neural networks with time delay in the leakage term and mixed delays: sample-data approach," Journal of the Egyptian Mathematical Society, vol. 24, no. 1, pp. 143-150, 2016.

[14] R. Samidurai, S. Rajavel, Q. Zhu, R. Raja, and H. Zhou, "Robust passivity analysis for neutral-type neural networks with mixed and leakage delays," Neurocomputing, vol. 175, pp. 635-643, 2016.

[15] B. Liu, "Global exponential stability for BAM neural networks with time-varying delays in the leakage terms," Nonlinear Analysis: Real World Applications, vol. 14, no. 1, pp. 559-566, 2013.

[16] X. Zhang and Q. Han, "State estimation for static neural networks with time-varying delays based on an improved reciprocally convex inequality," IEEE Transactions on Neural Networks and Learning Systems, pp. 1-6, 2017.

[17] J. Wang, X. Zhang, and Q. Han, "Event-triggered generalized dissipativity filtering for neural networks with time-varying delays," IEEE Transactions on Neural Networks and Learning Systems, vol. 27, no. 1, pp. 77-88, 2016.

[18] X. Zhang and Q. Han, "Global asymptotic stability analysis for delayed neural networks using a matrix-based quadratic convex approach," Neural Networks, vol. 54, pp. 57-69, 2014.

[19] K. Wang, Z. Teng, and H. Jiang, "Adaptive synchronization in an array of linearly coupled neural networks with reactiondiffusion terms and time delays," Communications in Nonlinear Science and Numerical Simulation, vol. 17, no. 10, pp. 3866-3875, 2012.

[20] Q. Gan, "Exponential synchronization of stochastic CohenGrossberg neural networks with mixed time-varying delays and reaction-diffusion via periodically intermittent control," Neural Networks, vol. 31, pp. 12-21, 2012.

[21] P. Wei, J. Wang, Y. Huang, B. Xu, and S. Ren, "Passivity analysis of impulsive coupled reaction-diffusion neural networks with and without time-varying delay," Neurocomputing, vol. 168, pp. 13-22, 2015.

[22] Y. Wang and J. Cao, "Exponential stability of stochastic higherorder BAM neural networks with reaction-diffusion terms and mixed time-varying delays," Neurocomputing, vol. 119, pp. 192200, 2013.

[23] J. Li, W. Zhang, and M. Chen, "Synchronization of delayed reaction-diffusion neural networks via an adaptive learning control approach," Computers and Mathematics with Applications, vol. 65, no. 11, pp. 1775-1785, 2013.

[24] K. Jing, "Exponential stability of FCNN with time-varying leakage delays and reaction-diffusion terms," Neurocomputing, vol. 145, pp. 363-368, 2014.

[25] C. Hu, J. Yu, H. Jiang, and Z. Teng, "Exponential synchronization for reaction-diffusion networks with mixed delays in terms of p-norm via intermittent driving," Neural Networks, vol. 31, pp. 1-11, 2012.

[26] K. Wang, Stochastic Biomathematics Model, The Science Publishing Company, Beijing, 2010.

[27] J. Mei, M. Jiang, B. Wang, Q. Liu, W. Xu, and T. Liao, "Exponential synchronization of non-autonomous cohen-grossberg neural networks with reaction-diffusion terms via periodically intermittent control," Neural Processing Letters, vol. 40, no. 2, pp. 103-126, 2014.

[28] X. Mao, Stochastic differential equations and applications, Chichester: Horwood Publication, 2nd edition, 2007.

[29] C. Gu, D. Li, S. Chen, S. Zheng, and Y. Tan, Equations of Mathematical Physics, Beijing: Higher Education Press, 2002.

[30] J. Pan, X. Liu, and S. Zhong, "Stability criteria for impusive reaction-diffusion Cohen-Grossberg neural networks with time-varying delays," Mathematical and Computer Modelling, vol. 51, pp. 1037-1050, 2010.

[31] H. Gu, "Adaptive synchronization for competitive neural networks with different time scales and stochastic perturbation," Neurocomputing, vol. 73, pp. 350-356, 2009. 


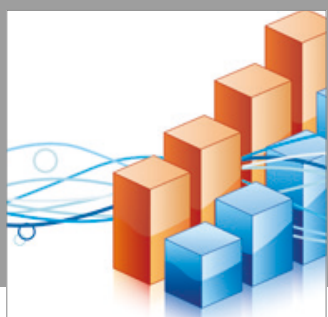

Advances in

Operations Research

vatersals

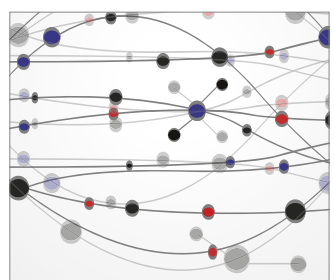

\section{The Scientific} World Journal
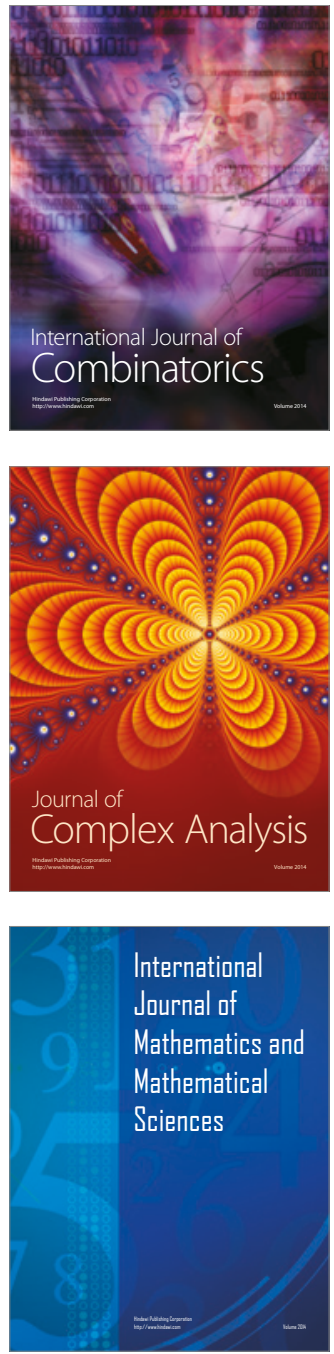
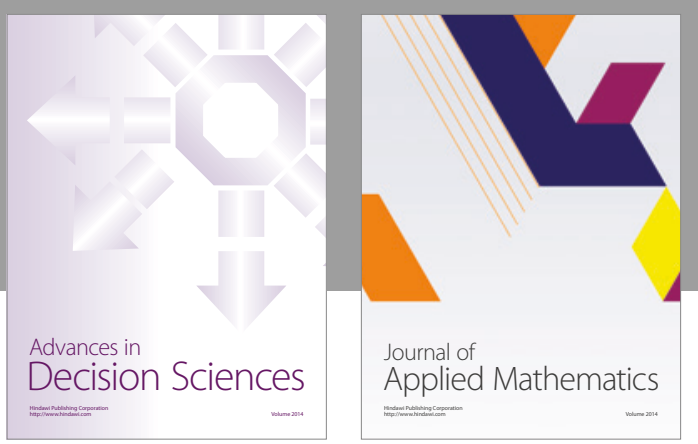

Algebra

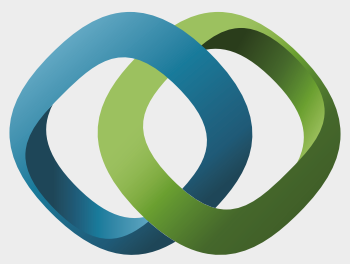

\section{Hindawi}

Submit your manuscripts at

https://www.hindawi.com
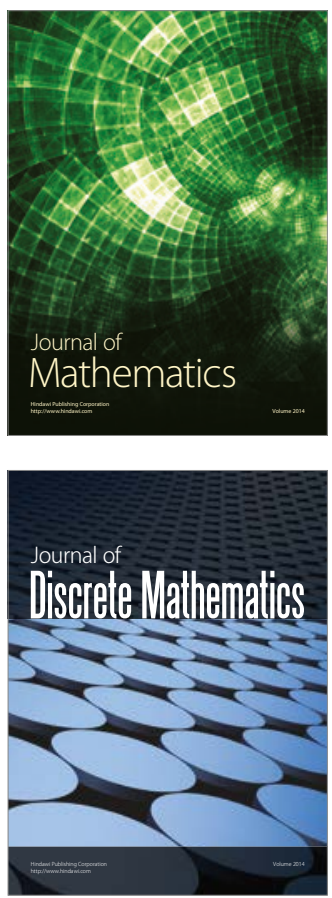

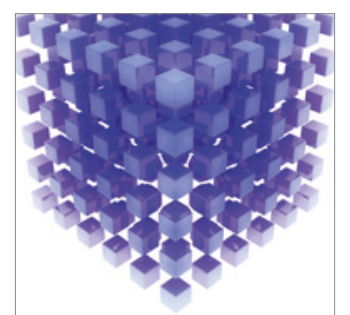

Mathematical Problems in Engineering
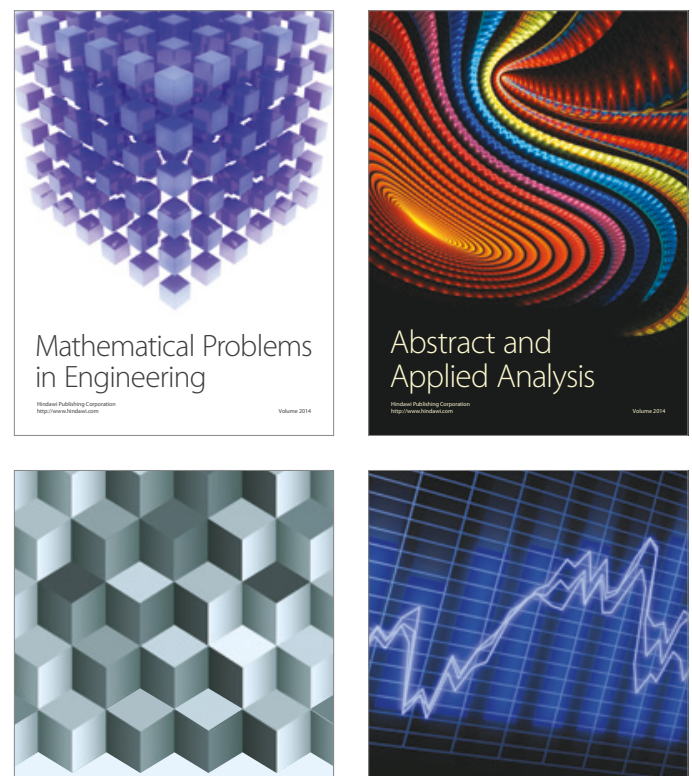

Journal of

Function Spaces

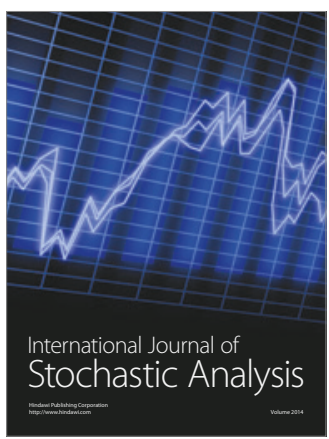

Probability and Statistics
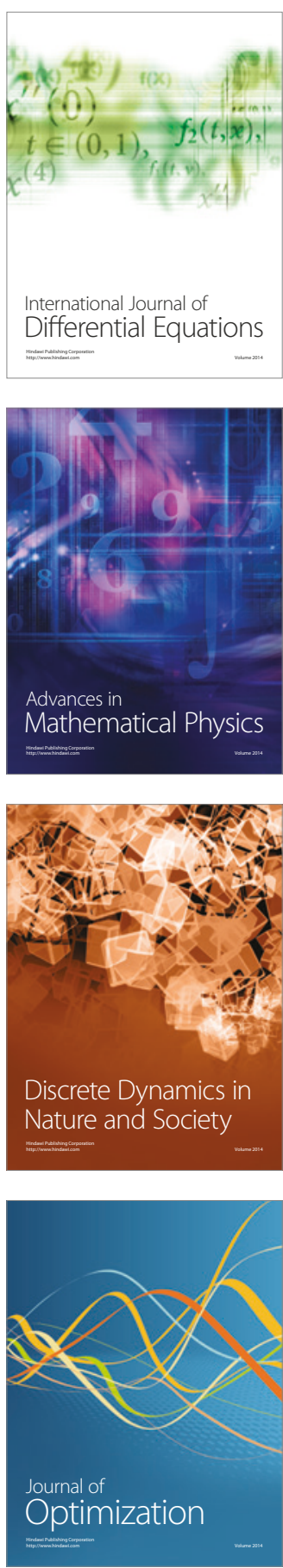